\title{
DEFINABILITY VIA KALIMULLIN PAIRS IN THE STRUCTURE OF THE ENUMERATION DEGREES
}

\author{
HRISTO A. GANCHEV AND MARIYA I. SOSKOVA
}

\begin{abstract}
We give an alternative definition of the enumeration jump operator. We prove that the class of total enumeration degrees and the class of low enumeration degrees are first order definable in the local substructure of the enumeration degree, consisting of the elements bounded by $\mathbf{0}_{e}{ }^{\prime}$.
\end{abstract}

\section{INTRODUCTION}

The main focus in degree theory, established as one of the core areas in computability theory, is to understand a mathematical structure, which arises as a formal way of classifying the computational strength of an object. The most studied examples of such structures are that of the Turing degrees, $\mathcal{D}_{T}$, based on the notion of Turing reducibility, as well as its local substructures, of the Turing degrees reducible to the first jump of the least degree, $\mathcal{D}_{T}\left(\leq \mathbf{0}_{T}{ }^{\prime}\right)$, and of the computably enumerable degrees, $\mathcal{R}$. In investigating such a mathematical structure, among the main questions that we ask is: how complex is this structure? The complexity of a structure can be inspected from many different aspects: how rich is it algebraically? how complicated is its theory? what sets are definable in it? does it have nontrivial automorphisms? The question about definability, in particular, is interrelated with all of the other questions and can be seen as a key to understanding the natural concepts that are approximated by the corresponding mathematical formalism. Research of the Turing degrees has been successful in providing a variety of results on definability. For the global theory of the Turing degrees, among the most notable results is the definability of the jump operator by Slaman and Shore [20]. The method used in the proof of this result, as well as many other definability results in $\mathcal{D}_{T}$, leads Slaman and Woodin to conjecture that every definable set in second order arithmetic is definable in $\mathcal{D}_{T}$. This is a consequence of their biinterpretability conjecture, which is shown to be equivalent to the rigidity of $\mathcal{D}_{T}[21$.

In the local theory Nies, Shore and Slaman [15. show a weakening of the biinterpretability conjecture for the computably enumerable degrees and obtain from it the first order definition of the jump classes $H_{n}$ and $L_{n+1}$ in $\mathcal{R}$, for every natural number $n$. Recently Shore [18] has shown that the same weakening of the

Received by the editors November 12, 2012 and, in revised form, April 10, 2013.

2010 Mathematics Subject Classification. Primary 03D30.

Key words and phrases. Enumeration reducibility, first order definability, enumeration jump, total enumeration degrees, low enumeration degrees.

This research was supported by a BNSF grant No. DMU 03/07/12.12.2011 and by Sofia University SF grant No. 131/09.05.2012.

The second author was supported by a Marie Curie international outgoing fellowship STRIDE (298471) within the 7th European Community Framework Programme and the Isaac Newton Institute for Mathematical Sciences in the programme 'Semantics and Syntax'. 
biinterpretability conjecture holds in the $\Delta_{2}^{0}$ Turing degrees, and so the classes $H_{n}$ and $L_{n+1}$ for every natural number $n$ are definable in $\mathcal{D}_{T}\left(\leq \mathbf{0}_{T}{ }^{\prime}\right)$ as well. One class of degrees which has managed to elude every attempt at definability in both local structures is that of the low $w_{1}$ degrees, $L_{1}$, the degrees whose jump is the least possible degree, $\mathbf{0}_{T}{ }^{\prime}$.

Another approach for understanding a structure, often used in mathematics, is to place this structure in a richer context, a context which would reveal new hidden relationships. The most promising candidate for such a larger context is the structure of the enumeration degrees, introduced by Friedberg and Rogers [5]. This structure is induced by a weaker form of relative computability: a set $A$ is enumeration reducible to a set $B$ if every enumeration of the set $B$ can be effectively transformed into an enumeration of the set $A$. The induced structure of the enumeration degrees, $\mathcal{D}_{e}$, is an upper-semilattice with jump operation and least element. The Turing degrees can be embedded in the enumeration degrees via the standard embedding $\iota$ which maps the Turing degree of a set $A$ to the enumeration degree of $A \oplus \bar{A}$. This embedding preserves the order, the least upper bound and the jump operation. The range of $\iota$ is therefore a substructure of $\mathcal{D}_{e}$, which is isomorphic to $\mathcal{D}_{T}$. This structure will be denoted by $\mathcal{T} \mathcal{O} \mathcal{T}$, and its elements will be called the total enumeration degrees. An important question which immediately arises in this context, first set by Rogers [16, is whether $\mathcal{T O} \mathcal{T}$ is first order definable in $\mathcal{D}_{e}$. Rozinas [17 proves that every enumeration degree is the greatest lower bound of two total enumeration degrees; thus the total enumeration degrees are an automorphism base for $\mathcal{D}_{e}$. This gives further motivation for studying the issue of the definability of $\mathcal{T O} \mathcal{T}$ in $\mathcal{D}_{e}$, as it would provide a strong link between the automorphism problem for the structures of the Turing degrees and the enumeration degrees. If $\mathcal{T O} \mathcal{T}$ is definable in $\mathcal{D}_{e}$, then a nontrivial automorphism of $\mathcal{D}_{e}$ would yield a nontrivial automorphism of $\mathcal{D}_{T}$.

Definability in the enumeration degrees has had its successes as well. Kalimullin [12] has shown that the enumeration jump is definable in $\mathcal{D}_{e}$. The method used in his proof is significantly less complex than that used to prove the corresponding result in the Turing degrees. The definition of the enumeration jump is closer to the much sought natural definition (see Shore [19]), and is based on the first order definability of a relativized version of the notion of a Kalimullin pair, $\mathcal{K}$-pair. Here we will give an alternative proof of the definability of the enumeration jump which does not use relativization and we see as more natural in a sense that will be made precise.

The jump operation gives rise to a local structure in the enumeration degrees, $\mathcal{G}_{e}$, consisting of all enumeration degrees that are reducible to the first jump, $\mathbf{0}_{e}{ }^{\prime}$, of the least degree, $\mathbf{0}_{e}$. As $\iota$ preserves the jump operation, it follows that $\mathcal{T} \mathcal{O} \mathcal{T} \cap \mathcal{G}_{e}$ is a structure which is isomorphic to $\mathcal{D}_{T}\left(\leq \mathbf{0}_{T}{ }^{\prime}\right)$, the local structure of the Turing degrees. In [6] we have shown that $\mathcal{K}$-pairs are first order definable in $\mathcal{G}_{e}$, providing the first step in the investigation of the definability theme for the local structure of the enumeration degrees. The local definition of $\mathcal{K}$-pairs unlocked numerous further results in the study of $\mathcal{G}_{e}$. For example in [6] we show that the classes of the upwards properly $\Sigma_{2}^{0}$ enumeration degrees and the downwards properly $\Sigma_{2}^{0}$ enumeration degrees are first order definable in $\mathcal{G}_{e}$, and in [8] we show that the first order theory of true arithmetic can be interpreted in $\mathcal{G}_{e}$, using coding methods based on $\mathcal{K}$-pairs. 
In this article we give two more examples of sets of degrees with natural first order definitions in $\mathcal{G}_{e}$. The first one gives a positive answer to the local version of Rogers' question. We show that the set of total $\Sigma_{2}^{0}$ enumeration degrees is first order definable in $\mathcal{G}_{e}$.

The second example supplies further evidence that studying the structure of the Turing degrees within the larger context of the enumeration degrees can provide us with more insight. We show that the set of low enumeration degrees is first order definable in $\mathcal{G}_{e}$. Combined with the local definability of the total enumeration degrees this gives the first instance of a local first order definition of an isomorphic copy of the low Turing degrees.

\section{Preliminaries}

We refer to Cooper [3] for a survey of basic results on the structure of the enumeration degrees and to Sorbi [22] for a survey of basic results on the local structure $\mathcal{G}_{e}$. We outline here basic definitions and properties of the enumeration degrees used in this article.

Definition 1. A set $A$ is enumeration reducible $\left(\leq_{e}\right)$ to a set $B$ if there is a c.e. set $\Phi$ such that

$$
A=\Phi(B)=\left\{n \mid \exists u\left(\langle n, u\rangle \in \Phi \& D_{u} \subseteq B\right)\right\},
$$

where $D_{u}$ denotes the finite set with code $u$ under the standard coding of finite sets. We will refer to the c.e. set $\Phi$ as an enumeration operator.

A set $A$ is enumeration equivalent $\left(\equiv_{e}\right)$ to a set $B$ if $A \leq_{e} B$ and $B \leq_{e} A$. The equivalence class of $A$ under the relation $\equiv_{e}$ is the enumeration degree $\mathbf{d}_{e}(A)$ of $A$. The structure of the enumeration degrees $\left\langle\mathcal{D}_{e}, \leq\right\rangle$ is the class of all enumeration degrees with relation $\leq$ defined by $\mathbf{d}_{e}(A) \leq \mathbf{d}_{e}(B)$ if and only if $A \leq_{e} B$. It has a least element $\mathbf{0}_{e}=\mathbf{d}_{e}(\emptyset)$, the set of all c.e. sets. We can define a least upper bound operation by setting $\mathbf{d}_{e}(A) \vee \mathbf{d}_{e}(B)=\mathbf{d}_{e}(A \oplus B)$.

The enumeration jump of a set $A$ is defined by Cooper [2].

Definition 2. The enumeration jump of a set $A$ is denoted by $J_{e}(A)$ and is defined as $K_{A} \oplus \overline{K_{A}}$, where $K_{A}=\left\{\langle e, x\rangle \mid e \in \mathbb{N} \& x \in \Phi_{e}(A)\right\}$. The enumeration jump of the enumeration degree of a set $A$ is $\mathbf{d}_{e}(A)^{\prime}=\mathbf{d}_{e}\left(J_{e}(A)\right)$.

By iterating the jump operation, we define inductively the $n$-th jump of a degree a for every $n: \mathbf{a}^{0}=\mathbf{a}$ and $\mathbf{a}^{n+1}=\left(\mathbf{a}^{n}\right)^{\prime}$.

Definition 3. A set $A$ is called total if $A \equiv_{e} A \oplus \bar{A}$. An enumeration degree is called total if it contains a total set. The collection of all total degrees is denoted by $\mathcal{T O} \mathcal{T}$.

As noted above, the structure $\mathcal{T O} \mathcal{T}$ is an isomorphic copy of the Turing degrees. The map $\iota$, defined by

$$
\iota\left(\mathbf{d}_{T}(A)\right)=\mathbf{d}_{e}(A \oplus \bar{A}),
$$

is an embedding of $\mathcal{D}_{T}$ in $\mathcal{D}_{e}$ which preserves the order, the least upper bound and the jump operation.

The local structure of the enumeration degrees, denoted by $\mathcal{G}_{e}$, is the substructure with domain, consisting of all enumeration degrees, which are reducible to $\mathbf{0}_{e}{ }^{\prime}$. As noted above, the elements of $\mathcal{G}_{e}$ are the enumeration degrees which contain $\Sigma_{2}^{0}$ sets, or equivalently, which consist entirely of $\Sigma_{2}^{0}$ sets. 
Definition 4. A set $A$ will be called low if $J_{e}(A) \equiv_{e} J_{e}(\emptyset)$. An enumeration degree $\mathbf{a} \in \mathcal{G}_{e}$ is low if $\mathbf{a}^{\prime}=\mathbf{0}_{e}{ }^{\prime}$.

\section{SEMI-RECURSIVE SETS}

In this section we will examine the properties of semi-recursive sets in the context of enumeration reducibility. This analysis extends the one that can be found in Arslanov, Cooper and Kalimullin [1].

Definition 5. We say that a set of natural numbers $A$ is semi-recursive if there is a total computable selector function $s_{A}: \mathbb{N} \times \mathbb{N} \rightarrow \mathbb{N}$, such that for any $x, y \in \mathbb{N}$, $s_{A}(x, y) \in\{x, y\}$ and whenever $\{x, y\} \cap A \neq \emptyset, s_{A}(x, y) \in A$.

Jockusch [1] showed that every nonzero Turing degree contains a semi-recursive set $A$ such that both $A$ and $\bar{A}$ are not c.e. In the context of enumeration reducibility this property can be translated as follows. A nonzero enumeration degree $\mathbf{t}$ is total if and only if there is a semi-recursive set $A$ which is not c.e. or co-c.e. such that

$$
\mathbf{t}=\mathbf{d}_{e}(A) \vee \mathbf{d}_{e}(\bar{A}) .
$$

Thus if we could show that the pairs of enumeration degrees of a semi-recursive set and its complement are first order definable, then we would be able to define the class of total enumeration degrees. This fact motivates the study of the structural properties of semi-recursive sets.

Our first discovery is that we can limit our investigations of semi-recursive sets to the study of semi-recursive sets of a particular kind. We describe these below.

Let $A$ be a set of natural numbers. We view the characteristic function of $A$ as an infinite binary string $\chi_{A}$, the $(n+1)$-th element of which, $\chi_{A}(n)$, is determined by the membership of $n$ in $A$. Namely, $\chi_{A}(n)=0$ if $n \notin A$ and $\chi_{A}(n)=1$ if $n \in A$. We will denote by $\chi\left\lceil n\right.$ the initial segment of $\chi_{A}$ of length $n$.

Finite binary strings are naturally ordered by the lexicographical ordering. Let $\sigma$ and $\rho$ be finite binary strings. We say that $\sigma$ is to the left of $\rho$, denoted by $\sigma<_{L} \rho$, if there is a finite binary string $\tau$ such that $\tau * 0 \subseteq \sigma$ and $\tau * 1 \subseteq \rho$. We can extend this relation to make it reflexive and linear as follows: $\sigma \leq_{L} \rho$ if $\sigma \subseteq \rho$ or $\sigma<_{L} \rho$.

Denote the length of a finite binary string $\sigma$ by $|\sigma|$. If $\sigma$ is a finite binary string and $\chi$ is an infinite binary string, then $\sigma \leq_{L} \chi$ and $\sigma<_{L} \chi$ will be shorthand for $\sigma \leq_{L} \chi \uparrow|\sigma|$ and $\sigma<_{L} \chi \uparrow|\sigma|$ respectively. The set of all finite binary strings to the left of or along $\chi_{A}$ will be denoted by $L_{A}$ :

$$
L_{A}=\left\{\sigma \in 2^{<\omega} \mid \sigma \leq_{L} \chi_{A}\right\} .
$$

The complement of $L_{A}$ will be denoted by $R_{A}$. Note that $R_{A}$ can be described as follows:

$$
R_{A}=\overline{L_{A}}=\left\{\sigma \in 2^{<\omega}\left|\sigma \not_{L} \chi_{A} \uparrow\right| \sigma \mid\right\}=\left\{\sigma \in 2^{<\omega}\left|\chi_{A} \uparrow\right| \sigma \mid<_{L} \sigma\right\} .
$$

Both sets $L_{A}$ and $R_{A}$ are easily shown to be semi-recursive, as the relation $\leq_{L}$ is a computable linear ordering on finite binary strings. The selector function $s_{L_{A}}$ simply outputs the smaller, with respect to $\leq_{L}$, of its two arguments. In the context of enumeration reducibility, the following properties make the pair $\left\{L_{A}, R_{A}\right\}$ particularly useful. 
Proposition 1. For every set of natural numbers $A$ the following hold:

(1) $L_{A} \leq_{e} A$;

(2) $R_{A} \leq_{e} \bar{A}$;

(3) $L_{A} \oplus R_{A} \equiv_{e} A \oplus \bar{A}$;

(4) $A$ is semi-recursive if and only if $A \leq_{1} L_{A}$, i.e. if there is a total computable injective function $g$ such that $n \in A$ if and only if $g(n) \in L_{A}$.

Proof. Let $A$ be a set of natural numbers. For every finite binary string $\sigma$ let $D_{\sigma}=\{n<|\sigma| \mid \sigma(n)=1\}$. We note here that if $D_{\sigma} \subseteq A$, then $\sigma \leq_{L} \chi_{A}$. If we assume otherwise: $\sigma \not_{L} \chi_{A}$, then there is a finite string $\tau$ of length $n$ which is a prefix of both $\sigma$ and $\chi_{A}$, and such that $\sigma(n)=1$ and $\chi(n)=0$. But then $n \in D_{\sigma} \backslash A$. Note that the reverse is not necessarily true.

(1) A finite string $\sigma$ is in $L_{A}$ if and only if there is a $\tau$, of the same length as $\sigma$, such that $\sigma \leq_{L} \tau$ and $D_{\tau} \subseteq A$. Indeed, if $\sigma \in L_{A}$ and $\sigma$ is of length $n$, then $\tau=\chi_{A} \uparrow(n+1)$ is such a string. If on the other hand $\sigma \leq_{L} \tau$ and $D_{\tau} \subseteq A$, then $\tau \leq_{L} \chi_{A}$, and hence by transitivity $\sigma \leq_{L} \chi_{A}$.

(2) Here it is worth noting that $R_{A} \cup\{\sigma \mid \sigma \subseteq A\}$ is 1-equivalent to $L_{\bar{A}}$ via the computable permutation which maps a finite string $\sigma$ to its mirror image $\bar{\sigma}$, inverting every bit. Thus $\sigma \in R_{A}$ if and only if there exists $\tau$ such that $\bar{\sigma}<_{L} \tau$ and $D_{\tau} \subseteq \bar{A}$.

(3) From (1) and (2) it follows that $L_{A} \oplus R_{A} \leq_{e} A \oplus \bar{A}$. On the other hand for every natural number $n, n \in \bar{A}$ if and only if there is a string $\sigma$ of length $n$ such that $\sigma * 0 \in L_{A}$ and $\sigma * 1 \in R_{A}$. Finally $n \in A$ if and only if there is a string $\sigma$ of length $n+1$ ending in 1 in $L_{A}$ such that for every string $\tau$ of length $n+1$, if $\sigma<_{L} \tau$, then $\tau \in R_{A}$.

(4) Suppose $A$ is semi-recursive with a selector function $s_{A}$. Then for every $n$ we build a string $\sigma_{n}$ of length $n+1$, ending in 1 , as follows. For every $m<n$ :

$$
\sigma_{n}(m)=\left\{\begin{array}{l}
0 \text { if } s_{A}(n, m)=n, \\
1 \text { if } s_{A}(n, m)=m .
\end{array}\right.
$$

We claim that $n \in A$ if and only if $\sigma_{n} \in L_{A}$. Suppose that $n \in A$; then for every $m \leq n, \sigma_{n}(m)=1$ implies $m \in A$ by the properties of the selector function. So if $n \in A$, then $D_{\sigma_{n}} \subseteq A$ and hence $\sigma_{n} \leq_{L} \chi_{A}$.

If $n \notin A$, then $\sigma_{n}(m)=0$ implies $m \in \bar{A}$ by the properties of the selector function. Thus $D_{\overline{\sigma_{n}}} \subseteq \bar{A}$ and hence $\overline{\sigma_{n}} \in L_{\bar{A}}$. Here $\overline{\sigma_{n}}$ is, as in (2), the mirror image of $\sigma_{n}$. Thus $\sigma_{n} \in R_{A} \cup\left\{\sigma \mid \sigma \subseteq \chi_{A}\right\}$. As $\sigma_{n}(n)=1$ and $n \notin A$, it follows that $\sigma_{n} \nsubseteq \chi_{A}$, so $\sigma_{n} \in R_{A}=\overline{L_{A}}$. This shows that $A \leq_{1} L_{A}$ via the function $g$, defined by $g(n)=\sigma_{n}$.

On the other hand if $A \leq_{1} L_{A}$ via a computable injective function $g$, then we can construct the selector function for $A$ using $g$ and the selector function for $L_{A}$ :

$$
s_{A}(x, y)=g^{-1}\left(s_{L_{A}}(g(x), g(y))\right) .
$$

We note here that the first three properties are in fact a proof of Jockusch's theorem for all sets $A$ whose Turing degree is not computably enumerable. The fourth property in Proposition 1 shows that up to enumeration equivalence all semi-recursive sets can be regarded as being of the form $L_{A}$ for some $A$. 
In terms of structure, the enumeration degrees of a semi-recursive $A$ and its complement behave as a minimal pair in a very strong sense. Arslanov, Cooper and Kalimullin [1] showed that for every set of natural numbers $X$ :

$$
\left(\mathbf{d}_{e}(A) \vee \mathbf{d}_{e}(X)\right) \wedge\left(\mathbf{d}_{e}(\bar{A}) \vee \mathbf{d}_{e}(X)\right)=\mathbf{d}_{e}(X) .
$$

Unfortunately this statement cannot be reversed. In fact the class of enumeration degrees for which the statement can be reversed brings us to the next topic: $\mathcal{K}$-pairs.

\section{4. $\mathcal{K}$-PAirs And the Definability of the EnUmeration JUmP}

In this section we will define the notion of a $\mathcal{K}$-pair, give examples of this notion, discuss basic properties and give an alternative first order definition of the enumeration jump.

Definition 6. Let $A$ and $B$ be sets of natural numbers. The pair $\{A, B\}$ is a Kalimullin pair $1 \mathcal{1}(\mathcal{K}$-pair) if there is a c.e. set $W$ such that

$$
A \times B \subseteq W \& \bar{A} \times \bar{B} \subseteq \bar{W} .
$$

As a first example of a $\mathcal{K}$-pair consider a c.e. set $U$ and an arbitrary set of natural numbers $A$. Then $U$ and $A$ form a $\mathcal{K}$-pair via the c.e. set $U \times \mathbb{N}$. $\mathcal{K}$-pairs of this sort are considered trivial and we will not be interested in them. A $\mathcal{K}$-pair $\{A, B\}$ is nontrivial if $A$ and $B$ are not c.e.

Nontrivial $\mathcal{K}$-pairs exist. As anticipated, if $A$ is semi-recursive, then $\{A, \bar{A}\}$ is a $\mathcal{K}$-pair. Indeed let $s_{A}$ be the selector function for $A$ and let

$$
\overline{s_{A}}(n, m)=\left\{\begin{array}{l}
n \text { if } s_{A}(n, m)=m, \\
m \text { if } s_{A}(n, m)=n
\end{array}\right.
$$

Now consider the c.e. set $W=\left\{\left(s_{A}(n, m), \overline{s_{A}}(n, m)\right) \mid n, m \in \mathbb{N}\right\}$ and notice that $A \times \bar{A} \subseteq W$ and $\bar{A} \times \overline{\bar{A}}=\bar{A} \times A \subseteq \bar{W}$.

4.1. Basic properties of $\mathcal{K}$-pairs. Kalimullin [12 has shown that the enumeration degrees of $\mathcal{K}$-pairs are precisely the degrees which satisfy the strong minimalpair property described in the previous section. He shows that the property of being a $\mathcal{K}$-pair is degree theoretic and first order definable in the global structure. A pair of sets $\{A, B\}$ is a $\mathcal{K}$-pair if and only if

$$
\forall \mathbf{x} \in \mathcal{D}_{e}\left[\mathbf{x}=\left(\mathbf{x} \vee \mathbf{d}_{e}(A)\right) \wedge\left(\mathbf{x} \vee \mathbf{d}_{e}(B)\right)\right] .
$$

Thus we can lift the notion of a $\mathcal{K}$-pair to the enumeration degrees. A pair of enumeration degrees $\mathbf{a}$ and $\mathbf{b}$ shall be called a $\mathcal{K}$-pair if every member of $\mathbf{a}$ forms a $\mathcal{K}$-pair with every member of $\mathbf{b}$. By $\mathcal{K}(\mathbf{a}, \mathbf{b})$ we will denote the first order formula, which is true of $\mathbf{a}$ and $\mathbf{b}$ if and only if they form a $\mathcal{K}$-pair.

Some additional properties of $\mathcal{K}$-pairs that will become important later are listed below. Proofs of these properties can be found in [12]; however for completeness we provide them here as well.

\footnotetext{
${ }^{1}$ Kalimullin's original term for this notion is e-ideal.
} 
Proposition 2. Let $A$ and $B$ be a nontrivial $\mathcal{K}$-pair.

(1) $A \leq_{e} \bar{B}$ and $\bar{A} \leq_{e} B \oplus J_{e}(\emptyset)$. Similarly $B \leq_{e} \bar{A}$ and $\bar{B} \leq_{e} A \oplus J_{e}(\emptyset)$.

(2) The class of enumeration degrees of sets that form a $\mathcal{K}$-pair with $A$ is an ideal.

(3) The enumeration degrees $\mathbf{d}_{e}(A)$ and $\mathbf{d}_{e}(B)$ are incomparable and quasiminimal; i.e. the only total degree bounded by either of them is $\mathbf{0}_{e}$.

Proof. Let $W$ be a c.e. set such that $A \times B \subseteq W$ and $\bar{A} \times \bar{B} \subseteq \bar{W}$.

(1) First we will show that $A$ is equal to the set $\mathcal{A}=\{a \mid\langle a, b\rangle \in W \& b \in \bar{B}\}$. That $A \subseteq \mathcal{A}$ follows from $\bar{A} \times \bar{B} \cap W=\emptyset$. For the reverse inclusion we use the fact that $B$ is not c.e. Suppose towards a contradiction that there is an element $a \in A \backslash \mathcal{A}$. Then it follows that for every $b$, if $\langle a, b\rangle \in W$, then $b \in B$. As $A \times B \subseteq W$, this yields $B=\{b \mid\langle a, b\rangle \in W\}$ and hence $B$ is c.e.

To show that $\bar{A} \leq_{e} B \oplus J_{e}(\emptyset)$ we use a symmetric argument to prove that $\bar{A}=\{a \mid\langle a, b\rangle \in \bar{W} \& b \in B\}$.

(2) Let $C$ be a set reducible to $B$ and let $\Gamma$ be such that $C=\Gamma(B)$. The index of a c.e. witness for the fact that $A$ and $C$ form a $\mathcal{K}$-pair can be uniformly obtained from an index of $W$ and an index of $\Gamma$. Consider the set $U=\{\langle a, c\rangle \mid \exists D(\langle c, D\rangle \in \Gamma \&\{a\} \times D \subseteq W)\}$. If $\langle a, c\rangle \in A \times C$, then either is an axiom $\langle c, D\rangle \in \Gamma$ such that $D \subseteq B$. Then $\{a\} \times D \subseteq A \times B \subseteq W$. If $a \in \bar{A}$ and for an axiom $\langle c, D\rangle \in \Gamma$ we have that $\{a\} \times D \subseteq W$, then as $\bar{A} \times \bar{B} \subseteq \bar{W}$, it follows that $D \subseteq B$ and hence $c \in C$. Thus $A \times C \subseteq U$ and $\bar{A} \times \bar{C} \subseteq \bar{U}$. This shows that the class of enumeration degrees of sets that form a $\mathcal{K}$-pair with $A$ is downwards closed.

Now suppose that $A$ and $C$ are a $\mathcal{K}$-pair via the c.e. set $U$. We show that $A$ forms a $\mathcal{K}$-pair with $B \oplus C$, and in this case as well the index of a c.e. set witnessing this is uniformly obtained from an index for $W$ and an index for $U$. Consider the set $V=\{\langle a, 2 b\rangle \mid\langle a, b\rangle \in W\} \cup\{\langle a, 2 c+1\rangle \mid\langle a, c\rangle \in U\}$. Then $A \times(B \oplus C) \subseteq V$ and $\bar{A} \times(\overline{B \oplus C}) \subseteq \bar{V}$.

(3) We show that $A$ and $B$ are indeed a minimal pair, and hence incomparable. Suppose $X$ is a set, reducible to both $A$ and $B$. Let $\Gamma$ be such that $X=$ $\Gamma(A)$ and $\Lambda$ be such that $X=\Lambda(B)$. Then $X=\left\{x \mid \exists D_{1} \cdot D_{2}\left(\left\langle x, D_{1}\right\rangle \in\right.\right.$ $\left.\left.\Gamma \&\left\langle x, D_{2}\right\rangle \in \Lambda \& D_{1} \times D_{2} \subseteq W\right)\right\}$.

Finally we show that $B$ is of quasiminimal degree. Let $X \oplus \bar{X}$ be a total set reducible to $B$. Then $X \oplus \bar{X}$ forms a $\mathcal{K}$-pair with $A$. Hence by the first property if $X \oplus \bar{X}$ is not c.e., then $A$ is enumeration reducible to $\overline{X \oplus \bar{X}}=\bar{X} \oplus X \equiv_{e} X \oplus \bar{X}$. This contradicts the incomparability of the two members in a $\mathcal{K}$-pair. Thus $X \oplus \bar{X}$ is c.e.

Let us consider what Proposition 2 means for $\mathcal{K}$-pairs in the local structure of the enumeration degrees. Suppose $A, B \leq_{e} J_{e}(\emptyset)$ are a nontrivial $\mathcal{K}$-pair. Recall that the enumeration jump of $A$ is defined as $J_{e}(A)=K_{A} \oplus \overline{K_{A}}$, where $K_{A}=$ $\left\{\langle e, x\rangle \mid e \in \mathbb{N} \& x \in \Phi_{e}(A)\right\} . K_{A}$ is enumeration equivalent to $A$, and hence by the second property $K_{A}$ forms a $\mathcal{K}$-pair with $B$. By the first property we have that $\overline{K_{A}} \leq_{e} B \oplus J_{e}(\emptyset)=J_{e}(\emptyset)$. Thus $J_{e}(A) \leq_{e} J_{e}(\emptyset)$ and so $A$ is low. Of course by symmetry, $B$ is also low. Thus nontrivial $\mathcal{K}$-pairs in the local structure are made up of low components. As every low set is $\Delta_{2}^{0}$ this gives us a nice way to approximate these sets. 
Let $A$ be a $\Sigma_{2}^{0}$ set of natural numbers. An approximation to $A$ is a uniformly computable sequence of finite sets $\mathcal{A}=\left\{A_{s}\right\}_{s<\omega}$ such that for every natural number $n$ we have that $A(n)=\liminf A_{s}(n)$. If $A$ is $\Delta_{2}^{0}$, then by Shoenfield's Limit Lemma we can find an approximation $\mathcal{A}$ to $A$, called a $\Delta_{2}^{0}$ approximation, with the additional property that for every $n, \lim _{s} A_{s}(n)$ exists. If $A$ is c.e., then it has a $\Sigma_{1}^{0}$ approximation $\mathcal{A}$, in which for every element $x$ there is at most one stage $s$ such that $A_{s}(n) \neq A_{s+1}(n)$.

Kalimullin $[12$ showed that $\mathcal{K}$-pairs in the local structure can also be characterized dynamically.

Lemma 1 (Kalimullin). A pair of non-c.e. $\Delta_{2}^{0}$ sets $\{A, B\}$ is a $\mathcal{K}$-pair if and only if there are $\Delta_{2}^{0}$ approximations $\left\{A_{i}\right\}_{i<\omega}$ to $A$ and $\left\{B_{i}\right\}_{i<\omega}$ to $B$ such that

$$
\forall i\left(A_{i} \subseteq A \vee B_{i} \subseteq B\right) .
$$

Approximations with the property above will be called $\mathcal{K}$-approximations.

4.2. Definability of the enumeration jump. To show that the jump is definable, Kalimullin [12] introduces a relativized version of a $\mathcal{K}$-pair. If $U$ is a set of natural numbers, then $A$ and $B$ form a $\mathcal{K}$-pair over $U$ if there is a set $W \leq_{e} U$ such that $A \times B \subseteq W$ and $\bar{A} \times \bar{B} \subseteq \bar{W}$. His proof of the definability of $\mathcal{K}$-pairs also relativizes: a pair of sets $\{A, B\}$ is a $\mathcal{K}$-pair over a set $U$ if and only if

$$
\forall \mathbf{x} \in \mathcal{D}_{e}\left[\mathbf{x} \vee \mathbf{d}_{e}(U)=\left(\mathbf{x} \vee \mathbf{d}_{e}(U) \vee \mathbf{d}_{e}(A)\right) \wedge\left(\mathbf{x} \vee \mathbf{d}_{e}(U) \vee \mathbf{d}_{e}(B)\right)\right]
$$

A triple of degrees $\mathbf{a}, \mathbf{b}$ and $\mathbf{c}$ such that each pair $\{\mathbf{a}, \mathbf{b}\},\{\mathbf{a}, \mathbf{c}\}$ and $\{\mathbf{b}, \mathbf{c}\}$ is a nontrivial $\mathcal{K}$-pair (over $\mathbf{u}$ ) will be called a $\mathcal{K}$-triple (over $\mathbf{u}$ ). The first order definition of the enumeration jump given by Kalimullin is equivalent to the following. For every enumeration degree $\mathbf{u} \in \mathcal{D}_{e}, \mathbf{u}^{\prime}$ is the greatest enumeration degree which can be represented as the join of a $\mathcal{K}$-triple over $\mathbf{u}$.

Here we give an alternative definition of the enumeration jump, which does not use the relativized version of a $\mathcal{K}$-pair and is in that sense simpler. The proof of this result is for the most part an application of the properties of $\mathcal{K}$-pairs of the form $\left\{L_{A}, R_{A}\right\}$ for some $A$, discussed above.

Theorem 1. For every nonzero enumeration degree $\mathbf{u} \in \mathcal{D}_{e}, \mathbf{u}^{\prime}$ is the largest among all least upper bounds $\mathbf{a} \vee \mathbf{b}$ of nontrivial $\mathcal{K}$-pairs $\{\mathbf{a}, \mathbf{b}\}$, such that $\mathbf{a} \leq \mathbf{u}$.

Proof. First we observe that if the sets $A$ and $B$ form a nontrivial $\mathcal{K}$-pair, then $A \oplus B \leq_{e} J_{e}(A)$. This follows from the fact that $K_{A} \equiv_{e} A$, and so by the second property in Proposition 2, $K_{A}$ and $B$ form a nontrivial $\mathcal{K}$-pair. By the first property of Proposition 2, $B \leq_{e} \overline{K_{A}}$, so $A \oplus B \leq_{e} K_{A} \oplus \overline{K_{A}}=J_{e}(A)$.

Now fix a nonzero enumeration degree $\mathbf{u}$ and a nontrivial $\mathcal{K}$-pair $\{\mathbf{a}, \mathbf{b}\}$ such that $\mathbf{a} \leq \mathbf{u}$. Then $\mathbf{a} \vee \mathbf{b} \leq \mathbf{a}^{\prime} \leq \mathbf{u}^{\prime}$ by the monotonicity of the jump operation. This establishes the first direction of the proof.

The second direction must be split into two cases. Suppose that $\mathbf{u}$ is not low. Fix $U \in \mathbf{u}$. Then $L_{K_{U}} \oplus R_{K_{U}} \equiv_{e} K_{U} \oplus \overline{K_{U}} \equiv_{e} J_{e}(U)$ by the third property in Proposition 1 and $L_{K_{U}} \leq_{e} U$ by the first property in Proposition 11. As $U$ is not low and $R_{K_{U}}=\overline{L_{K_{U}}}$, neither of the two sets $L_{K_{U}}$ or $R_{K_{U}}$ is c.e. Let $\mathbf{l}=\mathbf{d}_{e}\left(L_{K_{U}}\right)$ and $\mathbf{r}=\mathbf{d}_{e}\left(R_{K_{U}}\right)$. Then $\{\mathbf{l}, \mathbf{r}\}$ is a nontrivial $\mathcal{K}$-pair such that $\mathbf{l} \leq \mathbf{u}$ and $\mathbf{l} \vee \mathbf{r}=\mathbf{u}^{\prime}$.

Now suppose $\mathbf{u}$ is low. Then we cannot guarantee that $L_{K_{U}}$ and $R_{K_{U}}$ form a nontrivial $\mathcal{K}$-pair. However here we can use Theorem 4 from [7, which proves that for every nonzero $\Delta_{2}^{0}$ enumeration degree $\mathbf{a}$ there exists a nontrivial $\Delta_{2}^{0} \mathcal{K}$-pair 
$\{\mathbf{b}, \mathbf{c}\}$ such that $\mathbf{a} \vee \mathbf{b}=\mathbf{b} \vee \mathbf{c}=\mathbf{0}_{e}{ }^{\prime}$. Now as every low enumeration degree is $\Delta_{2}^{0}$, we can apply this theorem to $\mathbf{u}$ and obtain a nontrivial $\mathcal{K}$-pair $\{\mathbf{b}, \mathbf{c}\}$ such that $\mathbf{u} \vee \mathbf{b}=\mathbf{b} \vee \mathbf{c}=\mathbf{0}_{e}{ }^{\prime}$. Finally we apply the property $\mathcal{K}(\mathbf{b}, \mathbf{c})$ to $\mathbf{u}$ and get

$$
\mathbf{u}=(\mathbf{b} \vee \mathbf{u}) \wedge(\mathbf{c} \vee \mathbf{u})=\mathbf{0}_{e}{ }^{\prime} \wedge(\mathbf{c} \vee \mathbf{u})=(\mathbf{c} \vee \mathbf{u}) ;
$$

hence $\mathbf{c} \leq \mathbf{u}$.

We note here that this definition has the disadvantage that it cannot be applied to define $\mathbf{0}_{e}{ }^{\prime}$ in a simple way. A definition of $\mathbf{0}_{e}{ }^{\prime}$ can still be obtained from the facts that $\mathbf{0}_{e}{ }^{\prime}$ is the least possible enumeration jump and there are nonzero low degrees.

An immediate corollary of the proof of the alternative definition of the enumeration jump operation is McEvoy's jump inversion theorem [14].

Corollary 1 (McEvoy). For every total degree $\mathbf{a} \geq \mathbf{0}_{e}{ }^{\prime}$ there is a quasiminimal enumeration degree $\mathbf{b}$ such that $\mathbf{b}^{\prime}=\mathbf{a}^{\prime}$.

\section{Maximal $\mathcal{K}$-Pairs and the local definability of the total Degrees}

Let us consider again the special case of a $\mathcal{K}$-pair given by a semi-recursive nonc.e. set and its non-c.e. complement, say $\{A, \bar{A}\}$. This $\mathcal{K}$-pair can be considered as a maximal $\mathcal{K}$-pair, in the sense that there is no $\mathcal{K}$-pair $\{C, D\}$ such that $A \leq_{e} C$ and $\bar{A} \leq_{e} D$ and one of these reductions is strict. Indeed suppose there were such a pair $\{C, D\}$, and suppose for definiteness that $\bar{A}<_{e} D$. By the second property of Proposition 2, as $A \leq_{e} C$ and $\{C, D\}$ is a $\mathcal{K}$-pair, $A$ would also form a $\mathcal{K}$-pair with $D$. By the first property of Proposition $2, D \leq_{e} \bar{A}$, contradicting the strong inequality $\bar{A}<_{e} D$.

Definition 7. We say that $\{A, B\}$ is a maximal $\mathcal{K}$-pair if for every $\mathcal{K}$-pair $\{C, D\}$ such that $A \leq_{e} C$ and $B \leq_{e} D$, we have $A \equiv_{e} C$ and $B \equiv_{e} D$.

We can restate Jockusch's theorem about the existence of semi-recursive sets once again as follows:

Corollary 2. Every nonzero total set is enumeration equivalent to the join of the components of a maximal $\mathcal{K}$-pair.

The first order definability of the total enumeration degrees would then follow if it were true that maximality is the additional structural property needed to capture $\mathcal{K}$-pairs of the form $\{A, \bar{A}\}$. If this were true, then we can further argue that the definition of the enumeration jump given by Theorem 1 is natural as follows:

Consider the relation c.e. in on the Turing degrees defined by: $\mathbf{x}$ is c.e. in $\mathbf{u}$ if there are sets $X \in \mathbf{x}$ and $U \in \mathbf{u}$ such that $X$ is c.e. in $U$.

Proposition 3. Let $\mathbf{x}$ and $\mathbf{u}$ be Turing degrees such that $\mathbf{u}$ is nonzero. Then $\mathbf{x}$ is c.e. in $\mathbf{u}$ if and only if there is a $\mathcal{K}$-pair $\{A, \bar{A}\}$ such that $\mathbf{d}_{e}(A) \leq_{e} \iota(\mathbf{u})$ and $\iota(\mathbf{x})=\mathbf{d}_{e}(A) \vee \mathbf{d}_{e}(\bar{A})$.

Proof. Suppose that $\mathbf{x}$ is c.e. in $\mathbf{u}$. Let $X \in \mathbf{x}$ and $U \in \mathbf{u}$ be sets such that $X$ is c.e. in $U . X$ is c.e. in $U$ if and only if $X \leq_{e} U \oplus \bar{U}$. Consider the $\mathcal{K}$-pair $\left\{L_{X}, R_{X}\right\}$. By Proposition 1, $L_{X} \leq_{e} X \leq_{e} U \oplus \bar{U}$ and $L_{X} \oplus R_{X} \equiv_{e} X \oplus \bar{X}$. Thus $\mathbf{d}_{e}\left(L_{X}\right) \leq \mathbf{d}_{e}(U \oplus \bar{U})=\iota(\mathbf{u})$ and $\mathbf{d}_{e}\left(L_{X}\right) \oplus \mathbf{d}_{e}\left(R_{X}\right)=\mathbf{d}_{e}(X \oplus \bar{X})=\iota(\mathbf{x})$.

Suppose $\iota(\mathbf{x})=\mathbf{d}_{e}(A) \vee \mathbf{d}_{e}(\bar{A})$ for some $\mathcal{K}$-pair $\{A, \bar{A}\}$ such that $\mathbf{d}_{e}(A) \leq_{e} \iota(\mathbf{u})$. Again let $X \in \mathbf{x}$ and $U \in \mathbf{u}$. Then $A \leq_{e} U \oplus \bar{U}$ and hence $A$ is c.e. in $U$. On the other hand $A \oplus \bar{A} \equiv_{e} X \oplus \bar{X}$; hence $A \equiv_{T} X$. Thus $\mathbf{x}$ is c.e. in $\mathbf{u}$. 
Thus if every maximal $\mathcal{K}$-pair is of the form $\{A, \bar{A}\}$ for some $A$, then the total degrees would be definable and the relation c.e. in on the nonzero total degrees would be definable. The definition of the enumeration given in Theorem 1 restricted to the total degrees can then be read as: $\mathbf{u}^{\prime}$ is the largest total enumeration degree which is c.e. in $\mathbf{u}$.

In the local structure of the enumeration degrees, we can implement this plan in full. Let us consider maximal $\mathcal{K}$-pairs whose members are bounded by $\mathbf{0}_{e}{ }^{\prime}$. The universal quantifier in the first order definition of $\mathcal{K}$-pairs makes it nontrivial to show that their definability is preserved when restricted to the local structure $\mathcal{G}_{e}$. In [6] we show that this is nevertheless true.

Theorem 2 ([6]). There is a first order formula $\mathcal{L} K$ such that for any $\Sigma_{2}^{0}$ sets $A$ and $B,\{A, B\}$ is a nontrivial $\mathcal{K}$-pair if and only if $\mathcal{G}_{e}=\mathcal{L} K\left(\mathbf{d}_{e}(A), \mathbf{d}_{e}(B)\right)$.

Thus to prove that the class of total degrees is first order definable in $\mathcal{G}_{e}$, it suffices to show that the join of every maximal $\mathcal{K}$-pair in $\mathcal{G}_{e}$ is enumeration equivalent to a total set. We prove something stronger. We prove that every nontrivial $\mathcal{K}$-pair $\{A, B\}$ can be extended to a maximal $\mathcal{K}$-pair of the form $\{C, \bar{C}\}$. Recall that $\mathcal{K}$-pairs in the local structure consist of $\Delta_{2}^{0}$ sets.

Theorem 3. For every nontrivial $\Delta_{2}^{0} \mathcal{K}$-pair $\{A, B\}$ there is a $\Delta_{2}^{0} \mathcal{K}$-pair $\{C, \bar{C}\}$ such that $A \leq_{e} C$ and $B \leq_{e} \bar{C}$.

The proof of Theorem 3 is presented below. We can now combine Corollary 2 , Theorem 2 and Theorem 3 to establish:

Theorem 4. The set of total $\Sigma_{2}^{0}$ enumeration degrees is first order definable in $\mathcal{G}_{e}$.

Proof. Consider the formula:

$$
\begin{gathered}
\mathcal{T O T}(\mathbf{x}) \Longleftrightarrow \mathbf{x}=\mathbf{0}_{e} \vee \exists \mathbf{a} \exists \mathbf{b}[\mathcal{L} K(\mathbf{a}, \mathbf{b}) \& \mathbf{x}=(\mathbf{a} \vee \mathbf{b}) \& \\
\forall \mathbf{c} \forall \mathbf{d}(\mathcal{L} K(\mathbf{c}, \mathbf{d}) \& \mathbf{c} \geq \mathbf{a} \& \mathbf{d} \geq \mathbf{b} \rightarrow \mathbf{c}=\mathbf{a} \& \mathbf{d}=\mathbf{b})] .
\end{gathered}
$$

A $\Sigma_{2}^{0}$ enumeration degree $\mathbf{x}$ is total if and only if $\mathcal{G}_{e}=\mathcal{T} \mathcal{O} \mathcal{T}(\mathbf{x})$.

5.1. Extending to maximal $\mathcal{K}$-pairs. We give the proof of Theorem 3 ,

Proof. Fix a nontrivial $\Delta_{2}^{0} \mathcal{K}$-pair $\{A, B\}$ and let $\left\{A_{i}\right\}_{i<\omega}$ and $\left\{B_{i}\right\}_{i<\omega}$ be their respective $\Delta_{2}^{0} \mathcal{K}$-approximations. We build two $\Sigma_{2}^{0}$ sets $C$ and $D$ which will satisfy the following requirements:

(R1) $A=\{x \mid \exists j[2\langle x, j\rangle \in C]\}, B=\{x \mid \exists j[2\langle x, j\rangle+1 \in D]\}$;

(R2) $C$ and $D$ are $\Delta_{2}^{0}$;

(R3) $\bar{C}=D$;

(R4) $\{C, D\}$ is a $\mathcal{K}$-pair.

To ensure that these requirements are met, we construct respective $\Sigma_{2}^{0}$ approximations $\left\{C_{i}\right\}_{i<\omega}$ and $\left\{D_{i}\right\}_{i<\omega}$, which will have the following properties:

(P1) $A_{i}=\left\{x \mid \exists j\left[2\langle x, j\rangle \in C_{i}\right]\right.$ and $B_{i}=\left\{x \mid \exists j\left[2\langle x, j\rangle+1 \in D_{i}\right]\right.$. Assuming that the constructed approximations are $\Delta_{2}^{0}$ this will ensure that $A \supseteq\{x \mid$ $\exists j[2\langle x, j\rangle \in C]\}$ and $B \supseteq\{x \mid \exists j[2\langle x, j\rangle+1 \in D]\}$.

(P2) $\forall i\left[A_{i} \not \subset A \Rightarrow D_{i} \subseteq D\right]$ and $\forall i\left[B_{i} \not \subseteq B \Rightarrow C_{i} \subseteq C\right]$. This property will ensure that the constructed approximations are $\Delta_{2}^{0}$, i.e. (R2) holds. Indeed, if we assume that for some $d \notin D$ the set $I(d)=\left\{i \mid d \in D_{i}\right\}$ is infinite, then $\left\{A_{i}\right\}_{i \in I(d)}$ is a c.e. approximation to $A$, contradicting that $A$ is not 
c.e. Furthermore together with (P1) it ensures the inclusions $A \subseteq\{x \mid$ $\exists j[2\langle x, j\rangle \in C]\}$ and $B \subseteq\{x \mid \exists j[2\langle x, j\rangle+1 \in D]\}$. The argument is similar: there are infinitely many stages $i$ such that $B_{i} \nsubseteq B$; otherwise $B_{i}$ would turn out c.e. So for every $x \in A$ we can find a stage $i$ such that $x \in A_{i}$ and $B_{i} \nsubseteq \nsubseteq B$. By ( $\left.\mathrm{P} 1\right)$ there is a number $j$ such that $2\langle x, j\rangle \in C_{i}$. By (P2) $C_{i} \subseteq C$. The second inclusion is proved in a similar way.

(P3) $\forall i\left[C_{i} \cap D_{i}=\emptyset\right]$ and every natural number is eventually enumerated in one of the sets. This will ensure (R3).

(P4) $\forall i\left[C_{i} \subseteq C \vee D_{i} \subseteq D\right]$. This will ensure (R4).

Note that the property (P2) is a consequence of properties (P1) and (P4), so let us consider in more detail what the property (P4) is expressing. Suppose that $x \notin C$, but for some $i, x \in C_{i}$. Then $i$ is a bad stage for $C$, i.e. $C_{i} \nsubseteq C$, and we must ensure that all the elements in $D_{i}$ are ultimately enumerated in $D$. Thus from this point on the element $x$ is connected to all elements in $D_{i}$, in the sense that we should not enumerate $x$ in $D_{k}$ at a further stage $k>i$ unless we also ensure that $D_{i} \subseteq D_{k}$. This suggests the following relations for every stage $j$ :

$$
r_{j}(x, y) \Longleftrightarrow \exists i \leq j\left[x \in C_{i} \& y \in D_{i}\right]
$$

The main property (MP) of the construction is as follows: for every stage $j$ and every $x$ and $y$, if $r_{j}(x, y)$, then

$$
x \in D_{j} \Longrightarrow y \in D_{j} \text { and } y \in C_{j} \Longrightarrow x \in C_{j} .
$$

Note that (MP) automatically ensures that the two constructed approximations have the $\mathcal{K}$-pair property. The construction must therefore ensure that (P1), (P3) and (MP) are true. The other properties are implied by these.

5.1.1. Construction. We introduce the following piece of notation: with $\mathbf{c}_{i}^{a}$ we shall denote the natural number $2\langle a, i\rangle$, and by $\mathbf{d}_{i}^{b}$ the natural number $2\langle b, i\rangle+1$. If $a \in A_{i}$ we shall say that $\mathbf{c}_{i}^{a}$ is a follower of $a$, and similarly, if $b \in B_{i}$ we shall say that $\mathbf{d}_{i}^{b}$ is a follower of $b$. Note that by the properties of the construction we will have that $a \in A$ if and only if at least one of its followers is in $C$ and $b \in B$ if and only if at least one of its followers is in $D$. During the construction each follower will have one of the following two states: free or not free. Intuitively a follower is free if it is not currently enumerated in either of the sets $C$ or D. By Free we denote the set of all followers that are currently free.

The construction will be carried out in stages. Every stage consists of two parts, Extracting and Adding. We shall describe the construction formally and supply a brief description of the intuition for every action. The main intuition of the construction is that followers $\mathbf{c}_{j}^{a}$ want to end up in the set $D$ and followers $\mathbf{d}_{j}^{b}$ want to end up in the set $C$. A follower $\mathbf{c}_{j}^{a}$ remains in the set $C\left(\mathbf{d}_{j}^{b}\right.$ remains in the set $D$ ) only if it is forced to do so by other followers to which it is connected.

\section{Start of construction.}

We set $C_{0}=\left\{\mathbf{c}_{0}^{a} \mid a \in A_{0}\right\}$ and $D_{0}=\left\{\mathbf{d}_{0}^{b} \mid b \in B_{0}\right\}$. At stage $i>0$ we construct $C_{i}$ and $D_{i}$ by modifying $C_{i-1}$ and $D_{i-1}$ respectively as follows:

Initially we set $C_{i}=C_{i-1}$ and $D_{i}=D_{i-1}$.

\section{Part 1: Extracting}

(E1) For all $\mathbf{c}_{j}^{a} \in C_{i}$ such that $a \notin A_{i}$ we extract $\mathbf{c}_{j}^{a}$ from $C_{i}$. For all $\mathbf{d}_{j}^{b} \in D_{i}$ such that $b \notin B_{i}$ we extract $\mathbf{d}_{j}^{a}$ from $D_{i}$. 
Intuition: This action ensures that $\left\{a \mid \exists j\left[\mathbf{c}_{j}^{a} \in C_{i}\right]\right\} \subseteq A_{i}$ and that $\{b \mid$ $\left.\exists j\left[\mathbf{d}_{j}^{b} \in D_{i}\right]\right\} \subseteq B_{i}$.

(E2) For all $\mathbf{d}_{j}^{b} \in C_{i}$ such that $\left\{\mathbf{c}_{k}^{a} \mid r_{i-1}\left(\mathbf{c}_{k}^{a}, \mathbf{d}_{j}^{b}\right)\right\} \nsubseteq C_{i}$ we extract $\mathbf{d}_{j}^{b}$ from $C_{i}$. For all $\mathbf{c}_{j}^{a} \in D_{i}$ such that $\left\{\mathbf{d}_{k}^{b} \mid r_{i-1}\left(\mathbf{c}_{j}^{a}, \mathbf{d}_{k}^{b}\right)\right\} \nsubseteq D_{i}$ we extract $\mathbf{c}_{j}^{a}$ from $D_{i}$.

Intuition: The follower $\mathbf{d}_{j}^{b}$ is only allowed to remain in $C_{i}$ if all of the elements to which it has been connected at a previous stage, i.e. $\left\{\mathbf{c}_{k}^{a} \mid\right.$ $\left.r_{i-1}\left(\mathbf{c}_{k}^{a}, \mathbf{d}_{j}^{b}\right)\right\}$, are still in $C_{i}$. These elements we can consider as requested in $C_{i}$ by $\mathbf{d}_{j}^{b}$. If one of these requests cannot be fulfilled (due to the properties of $A_{i}$ for example and rule (E1)), $\mathbf{d}_{j}^{b}$ must also be extracted from $C_{i}$. Similar reasoning is applied to followers $\mathbf{c}_{j}^{a}$ and their membership to $D_{i}$. These actions ensure that the main property of the construction is true.

(E3) For all $\mathbf{c}_{j}^{a} \in C_{i}$ such that $\left\{\mathbf{d}_{k}^{b} \mid r_{i-1}\left(\mathbf{c}_{j}^{a}, \mathbf{d}_{k}^{b}\right)\right\} \cap C_{i}=\emptyset$ we extract $\mathbf{c}_{j}^{a}$ from $C_{i}$.

For all $\mathbf{d}_{j}^{b} \in D_{i}$ such that $\left\{\mathbf{c}_{k}^{a} \mid r_{i-1}\left(\mathbf{c}_{k}^{a}, \mathbf{d}_{j}^{b}\right)\right\} \cap D_{i}=\emptyset$ we extract $\mathbf{d}_{j}^{b}$ from $D_{i}$.

Intuition: A follower $\mathbf{c}_{j}^{a}$ was forced into $C_{i-1}$ because of a request by some $\mathbf{d}_{k}^{b}$ to which it is connected. However at this stage the follower that made this request is no longer in $C_{i}$ (it was extracted under (E2) as one of its other requests was not fulfilled). In other words $\mathbf{c}_{j}^{a}$ is not requested any longer in $C_{i}$, so it is free to leave and attempt entering $D_{i}$.

All extracted elements become free.

Part 2: Adding

(A1) For all free $\mathbf{d}_{j}^{b}$ such that $\left\{\mathbf{c}_{k}^{a} \mid r_{i-1}\left(\mathbf{c}_{k}^{a}, \mathbf{d}_{j}^{b}\right)\right\} \subseteq C_{i} \cup$ Free and $\left\{a \mid r_{i-1}\left(\mathbf{c}_{k}^{a}, \mathbf{d}_{j}^{b}\right)\right\}$ $\subseteq A_{i}$ we enumerate $\mathbf{d}_{j}^{b}$ and $\left\{\mathbf{c}_{k}^{a} \mid r_{i-1}\left(\mathbf{c}_{k}^{a}, \mathbf{d}_{j}^{b}\right)\right\}$ in $C_{i}$.

All enumerated elements become not free.

For all free $\mathbf{c}_{j}^{a}$ such that $\left\{\mathbf{d}_{k}^{b} \mid r_{i-1}\left(\mathbf{c}_{j}^{a}, \mathbf{d}_{k}^{b}\right)\right\} \subseteq D_{i} \cup$ Free and $\left\{b \mid r_{i-1}\left(\mathbf{c}_{j}^{a}, \mathbf{d}_{k}^{b}\right)\right\}$ $\subseteq B_{i}$ we enumerate $\mathbf{c}_{j}^{a}$ and $\left\{\mathbf{d}_{k}^{b} \mid r_{i-1}\left(\mathbf{c}_{j}^{a}, \mathbf{d}_{k}^{b}\right)\right\}$ in $D_{i}$.

All enumerated elements become not free.

Intuition: This is the action that allows followers $\mathbf{d}_{j}^{b}$ to enter $C_{i}$ and respectively $\mathbf{c}_{j}^{a}$ to enter $D_{i}$. This can be done only if all of their requests can be fulfilled at the same time. These requests must also not injure the actions of rule (E1).

(A2) For all $a \in A_{i}$ we enumerate $\mathbf{c}_{i}^{a}$ in $C_{i}$.

For all $b \in B_{i}$ we enumerate $\mathbf{d}_{i}^{b}$ in $D_{i}$.

All enumerated elements become not free.

Intuition: This action ensures that $\left\{a \mid \exists j\left[\mathbf{c}_{j}^{a} \in C_{i}\right]\right\} \supseteq A_{i}$ and that $\{b \mid$ $\left.\exists j\left[\mathbf{d}_{j}^{b} \in D_{i}\right]\right\} \supseteq B_{i}$ and together with (E1), property (P1).

(A3) For all $a, j \leq i$ such that $a \notin A_{j}$ we enumerate $\mathbf{c}_{j}^{a} \in D_{i}$. For all $b, j \leq i$ such that $b \notin B_{j}$ we enumerate $\mathbf{d}_{j}^{b} \in C_{i}$.

Intuition: This action handles elements that are not followers. As our aim is to construct $D$ as $\bar{C}$, these elements also need to be enumerated in one of the two constructed sets. Note that even elements are enumerated in $D_{i}$ and odd elements are enumerated in $C_{i}$. At the following stage an even number $\mathbf{c}_{j}^{a}$, which was enumerated in $D_{i}$ under this action, cannot be extracted 
under rules (E1) and (E3). Furthermore as $\mathbf{c}_{j}^{a}$ has never been enumerated into an approximating set to $C$, the set $\left\{\mathbf{d}_{k}^{a} \mid r_{i}\left(\mathbf{c}_{j}^{a}, \mathbf{d}_{k}^{b}\right)\right\}$ is empty, so it cannot be extracted under rule (E2). Thus this element remains in $D_{k}$ at all further stages $k>i$. Similar reasoning is applied to odd numbers, enumerated in $D_{i}$ under this action.

End of construction.

5.1.2. Verification of the construction. We prove that the described construction produces sets $C$ and $D$, which have the properties listed as (P1)-(P4) and (MP). We start with the easiest property: (P1).

Proposition 4. For every $i, A_{i}=\left\{a \mid \exists j\left[\mathbf{c}_{j}^{a} \in C_{i}\right]\right\}$ and $B_{i}=\left\{b \mid \exists j\left[\mathbf{d}_{j}^{b} \in D_{i}\right]\right\}$.

Proof. The claims of the proposition follow directly from rules (E1), (A1) and (A2). Indeed (A2) guarantees the inclusion $\subseteq$, as $A_{i}=\left\{a \mid \mathbf{c}_{i}^{a} \in C_{i}\right\}$ and $B_{i}=\left\{b \mid \mathbf{d}_{i}^{b} \in\right.$ $\left.D_{i}\right\}$. On the other hand (E1) and (A1) enforce that $A_{i} \supseteq\left\{a \mid \exists j\left[\mathbf{c}_{j}^{a} \in C_{i}\right]\right\}$ and $B_{i} \supseteq\left\{b \mid \exists j\left[\mathbf{d}_{j}^{b} \in D_{i}\right]\right\}$.

The following proposition is a direct consequence of the construction. We state it nevertheless for completeness.

Proposition 5. For all i, $C_{i} \cap D_{i}=\emptyset$.

Next we turn to the main property of the construction (MP). One particular case of it will be used frequently in the rest of the proof, and we will state and prove it here separately.

Proposition 6. If $\mathbf{c}_{j}^{a}$ is a follower and $\mathbf{c}_{j}^{a} \in D_{i}$ then $\left\{\mathbf{d}_{k}^{b} \mid r_{i}\left(\mathbf{c}_{j}^{a}, \mathbf{d}_{k}^{b}\right)\right\} \subseteq D_{i}$. If $\mathbf{d}_{j}^{b}$ is a follower and $\mathbf{d}_{j}^{b} \in C_{i}$, then $\left\{\mathbf{c}_{k}^{a} \mid r_{i}\left(\mathbf{c}_{k}^{a}, \mathbf{d}_{j}^{b}\right)\right\} \subseteq C_{i}$.

Proof. We prove the first statement. The second statement is proved similarly. Let $\mathbf{c}_{j}^{a}$ be a follower (i.e. $a \in A_{j}$ ) such that $\mathbf{c}_{j}^{a} \in D_{i}$. If $\mathbf{c}_{j}^{a}$ is enumerated in $D_{i}$ at stage $i$ under rule (A1), then by construction the set $\left\{\mathbf{d}_{k}^{b} \mid r_{i}\left(\mathbf{c}_{j}^{a}, \mathbf{d}_{k}^{b}\right)\right\}$ is also enumerated in $D_{i}$. As no more elements are extracted from $D_{i}$ after the execution of step (A1), it follows that $\left\{\mathbf{d}_{k}^{b} \mid r_{i}\left(\mathbf{c}_{j}^{a}, \mathbf{d}_{k}^{b}\right)\right\} \subseteq D_{i}$.

The other possibility is that $\mathbf{c}_{j}^{a} \in D_{i-1}$ and during stage $i, \mathbf{c}_{j}^{a}$ is not extracted from $D_{i}$. But then the prerequisites of rule (E2) are not valid for $\mathbf{c}_{j}^{a}$ at stage $i$, and hence before starting the execution of (E3) it is true that $\left\{\mathbf{d}_{k}^{b} \mid r_{i}\left(\mathbf{c}_{j}^{a}, \mathbf{d}_{k}^{b}\right)\right\} \subseteq D_{i}$. During the execution of (E3) it is the case that for every $\mathbf{d}_{k}^{b} \in\left\{\mathbf{d}_{k}^{b} \mid r_{i}\left(\mathbf{c}_{j}^{a}, \mathbf{d}_{k}^{b}\right)\right\}$, $\mathbf{c}_{j}^{a} \in\left\{\mathbf{c}_{l}^{\alpha} \mid r_{i-1}\left(\mathbf{c}_{l}^{\alpha}, \mathbf{d}_{k}^{b}\right)\right\} \cap D_{i}$. By (E3) this intersection must be empty in order to extract $\mathbf{d}_{k}^{b}$ from $D_{i}$, so none of the elements in $\left\{\mathbf{d}_{k}^{b} \mid r_{i}\left(\mathbf{c}_{j}^{a}, \mathbf{d}_{k}^{b}\right)\right\}$ are extracted from $D_{i}$ during the execution of (E3). Thus finally $\left\{\mathbf{d}_{k}^{b} \mid r_{i}\left(\mathbf{c}_{j}^{a}, \mathbf{d}_{k}^{b}\right)\right\} \subseteq D_{i}$.

We are now ready to prove the main property (MP).

Lemma 2 (Main Lemma). Let $x$ and $y$ be natural numbers such that $r_{i}(x, y)$, for some natural number $i$. Then the following two conditions are true:

(C1) $x \in D_{i} \Longrightarrow y \in D_{i}$.

(C2) $y \in C_{i} \Longrightarrow x \in C_{i}$.

Proof. The claim of the lemma is trivial when either $x$ or $y$ is not a follower, as every such element is only enumerated once under (A3) in its corresponding set and is never extracted. For followers $x$ and $y$ we shall consider three different cases. 
Case 1. $x=\mathbf{c}_{j}^{a}$ and $y=\mathbf{d}_{k}^{b}$. This is a direct consequence of Proposition 6 .

Case 2. $x=\mathbf{c}_{j}^{a}$ and $y=\mathbf{c}_{l}^{\alpha}$ (or $x=\mathbf{d}_{k}^{b}$ and $y=\mathbf{d}_{l}^{\beta}$ ). Let $s$ be the least natural number for which $r_{s}(x, y)$. We shall prove simultaneously claims $(\mathrm{C} 1),(\mathrm{C} 2)$ and that

$$
\left\{\mathbf{d}_{k}^{b} \mid r_{i}\left(y, \mathbf{d}_{k}^{b}\right)\right\} \subseteq\left\{\mathbf{d}_{k}^{b} \mid r_{i}\left(x, \mathbf{d}_{k}^{b}\right)\right\}
$$

by induction on $i \geq s$. For $i=s$ claims $(\mathrm{C} 1)$ and $(\mathrm{C} 2)$ are trivially true, as by the definition of the relation $r_{s}$ and the choice of $s$ we have $x \in C_{s}$ and $y \in D_{s}$. For claim (11) suppose that $\mathbf{d}_{k}^{b}$ is such that $r_{s}\left(y, \mathbf{d}_{k}^{b}\right)$. Since $y \in D_{s}$, Proposition 6 implies that $\mathbf{d}_{k}^{b} \in D_{s}$, and hence from $x \in C_{s}$ we obtain $r_{s}\left(x, \mathbf{d}_{k}^{b}\right)$.

Now let $i>s$. In order to prove (C1) suppose that $x \in D_{i}$. Then according to Proposition $6,\left\{\mathbf{d}_{k}^{b} \mid r_{i}\left(x, \mathbf{d}_{k}^{b}\right)\right\} \subseteq D_{i}$. Now using the induction hypothesis for (11) and that $\left\{\mathbf{d}_{k}^{b} \mid r_{i-1}\left(x, \mathbf{d}_{k}^{b}\right)\right\} \subseteq\left\{\mathbf{d}_{k}^{b} \mid r_{i}\left(x, \mathbf{d}_{k}^{b}\right)\right\}$ we obtain $\left\{\mathbf{d}_{k}^{b} \mid r_{i-1}\left(y, \mathbf{d}_{k}^{b}\right)\right\} \subseteq D_{i}$. As by Proposition 5 we have that $D_{i} \cap C_{i}=\emptyset$, it follows that at stage $i$ when we reach step (E3), $\left\{\mathbf{d}_{k}^{b} \mid r_{i}\left(y, \mathbf{d}_{k}^{b}\right)\right\} \cap C_{i}=\emptyset$, which implies that $y \notin C_{i}$. This means that if $y$ is not already in $D_{i}$, it is free during the execution of (A1) and we would enumerate it in $D_{i}$.

In order to prove $(\mathrm{C} 2)$ suppose that $y \in C_{i}$. Then there is a $\mathbf{d}_{k}^{b} \in C_{i}$ such that $r_{i-1}\left(y, \mathbf{d}_{k}^{b}\right)$, since otherwise $y$ would have been extracted under (E3). From the induction hypothesis for (11) we obtain that $r_{i-1}\left(x, \mathbf{d}_{k}^{b}\right)$ and hence $x \in C_{i}$ by Proposition 6 .

Finally let us prove (11). We consider two cases. First suppose that $y \notin C_{i}$. Then $\left\{\mathbf{d}_{k}^{b} \mid r_{i}\left(y, \mathbf{d}_{k}^{b}\right)\right\}=\left\{\mathbf{d}_{k}^{b} \mid r_{i-1}\left(y, \mathbf{d}_{k}^{b}\right)\right\}$. On the other hand $\left\{\mathbf{d}_{k}^{b} \mid r_{i-1}\left(x, \mathbf{d}_{k}^{b}\right)\right\} \subseteq\left\{\mathbf{d}_{k}^{b} \mid r_{i}\left(x, \mathbf{d}_{k}^{b}\right)\right\}$, and now the claim follows from the induction hypothesis. Secondly let $y \in C_{i}$. Then

$$
\left\{\mathbf{d}_{k}^{b} \mid r_{i}\left(y, \mathbf{d}_{k}^{b}\right)\right\}=\left\{\mathbf{d}_{k}^{b} \mid r_{i-1}\left(y, \mathbf{d}_{k}^{b}\right)\right\} \cup\left\{\mathbf{d}_{k}^{b} \mid \mathbf{d}_{k}^{b} \in D_{i}\right\} .
$$

On the other hand by $(\mathrm{C} 2)$ we have $x \in C_{i}$ and hence

$$
\left\{\mathbf{d}_{k}^{b} \mid r_{i}\left(x, \mathbf{d}_{k}^{b}\right)\right\}=\left\{\mathbf{d}_{k}^{b} \mid r_{i-1}\left(x, \mathbf{d}_{k}^{b}\right)\right\} \cup\left\{\mathbf{d}_{k}^{b} \mid \mathbf{d}_{k}^{b} \in D_{i}\right\},
$$

and again the claim follows from the induction hypothesis.

Case 3. $x=\mathbf{d}_{k}^{b}$ and $y=\mathbf{c}_{j}^{a}$. Let $s$ be again the least stage for which $r_{s}(x, y)$. In particular $x \in C_{s}$ and $y \in D_{s}$. We shall prove simultaneously (C1), (C2) and for all $i \geq s$ :

$$
\begin{gathered}
\left\{\mathbf{d}_{l}^{\beta} \mid r_{i}\left(y, \mathbf{d}_{l}^{\beta}\right)\right\} \subseteq\left\{\mathbf{d}_{l}^{\beta} \mid r_{i}\left(x, \mathbf{d}_{l}^{\beta}\right)\right\}, \\
\left\{\mathbf{c}_{l}^{\alpha} \mid r_{i}\left(\mathbf{c}_{l}^{\alpha}, x\right)\right\} \subseteq\left\{\mathbf{c}_{l}^{\alpha} \mid r_{i}\left(\mathbf{c}_{l}^{\alpha}, y\right)\right\}
\end{gathered}
$$

by induction on $i$. For $i=s$ claims $(\mathrm{C} 1)$ and $(\mathrm{C} 2)$ are trivial. In order to prove (2) suppose that $\mathbf{d}_{l}^{\beta}$ is such that $r_{s}\left(y, \mathbf{d}_{l}^{\beta}\right)$. Then according to Proposition 6, $\mathbf{d}_{l}^{\beta} \in D_{s}$ and hence $r_{s}\left(x, \mathbf{d}_{l}^{\beta}\right)$. The proof of (3) is analogous.

Now let $i>s$. In order to prove (C1) suppose that $x \in D_{i}$. Then according to steps (E3) and (A1) of the construction there is a $\mathbf{c}_{l}^{\alpha} \in D_{i}$ such that $r_{i-1}\left(\mathbf{c}_{l}^{\alpha}, x\right)$. The induction hypothesis for (3) implies $r_{i-1}\left(\mathbf{c}_{l}^{\alpha}, y\right)$. Now from claim (C1) of Case 2 and $\mathbf{c}_{l}^{\alpha} \in D_{i}$ we obtain $y \in D_{i}$. The proof of (C2) is analogous.

Now let us prove (2). Suppose that for some $\mathbf{d}_{l}^{\beta}, r_{i}\left(y, \mathbf{d}_{l}^{\beta}\right)$. We shall consider two cases. First suppose that $y \notin C_{i}$. Then it should be the case 
$r_{i-1}\left(y, \mathbf{d}_{l}^{\beta}\right)$, which together with the induction hypothesis implies $r_{i-1}\left(x, \mathbf{d}_{l}^{\beta}\right)$ and hence $r_{i}\left(x, \mathbf{d}_{l}^{\beta}\right)$. Now let $y \in C_{i}$. If $r_{i-1}\left(y, \mathbf{d}_{l}^{\beta}\right)$ we reason in the same way as above, so suppose that $r_{i-1}\left(y, \mathbf{d}_{l}^{\beta}\right)$ is not true. Then it should be the case $\mathbf{d}_{l}^{\beta} \in D_{i}$. On the other hand (C2) implies $x \in C_{i}$ and hence $r_{i}\left(x, \mathbf{d}_{l}^{\beta}\right)$.

Claim (3) is proved analogously.

Next we show that property $(\mathrm{P} 2)$ is true.

Proposition 7. For every $i$ the following hold:

- $A_{i} \not \subset A \Longrightarrow D_{i} \subseteq D$;

- $B_{i} \nsubseteq B \Longrightarrow C_{i} \subseteq C$.

Furthermore

- $a \in A_{i} \backslash A \Longrightarrow \mathbf{c}_{i}^{a} \in D$;

- $b \in B_{i} \backslash B \Longrightarrow \mathbf{d}_{i}^{b} \in C$.

Proof. Fix an $i$ such that $A_{i} \nsubseteq \nsubseteq A$ and let $a \in A_{i} \backslash A$. Consider the follower $\mathbf{c}_{i}^{a}$. According to (A2) $\mathbf{c}_{i}^{a} \in C_{i}$, so that for all $y \in D_{i}, r_{i}\left(\mathbf{c}_{i}^{a}, y\right)$ and hence $r_{j}\left(\mathbf{c}_{i}^{a}, y\right)$ for $j \geq i$. Let $s_{1}>i$ be the least stage, such that for all $j \geq s_{1}, a \notin A_{j}$ (such stage exists since $\left\{A_{i}\right\}_{i<\omega}$ is a $\Delta_{2}^{0}$ approximation). Then according to rule (E1) for each $j \geq s_{1}, \mathbf{c}_{i}^{a} \notin C_{j}$ and hence for $j \geq s_{1},\left\{\mathbf{d}_{k}^{b} \mid r_{j}\left(\mathbf{c}_{i}^{a}, \mathbf{d}_{k}^{b}\right)\right\} \cap C_{j}=\emptyset$. Thus for $j \geq s_{1}$, $\left\{\mathbf{d}_{k}^{b} \mid r_{j}\left(\mathbf{c}_{i}^{a}, \mathbf{d}_{k}^{b}\right)\right\} \subseteq D_{j} \cup$ Free. Now consider the set $\left\{b \mid \exists k\left[r_{j}\left(\mathbf{c}_{i}^{a}, \mathbf{d}_{k}^{b}\right)\right]\right\}$. Note that as for all $j \geq s_{1}, \mathbf{c}_{i}^{a} \notin C_{j}$, it follows that this set is finite and does not change. We claim that

$$
\left\{b \mid \exists k\left[r_{j}\left(\mathbf{c}_{i}^{a}, \mathbf{d}_{k}^{b}\right)\right]\right\} \subseteq B .
$$

Indeed, $r_{j}\left(\mathbf{c}_{i}^{a}, \mathbf{d}_{k}^{b}\right)$ implies that for some $l, \mathbf{c}_{i}^{a} \in C_{l}$ and $\mathbf{d}_{k}^{b} \in D_{l}$, and in the particular $a \in A_{l}$ and $b \in B_{l}$. Thus $A_{l} \nsubseteq A$, so that by our choice of $\mathcal{K}$-approximations to $A$ and $B$, it must be true that $B_{l} \subseteq B$ and hence $b \in B$.

Fix the least stage $s_{2} \geq s_{1}$ such that for all $j \geq s_{2},\left\{b \mid \exists k\left[r_{j}\left(\mathbf{c}_{i}^{a}, \mathbf{d}_{k}^{b}\right)\right]\right\} \subseteq B_{j}$ (such a stage exists by virtue of (40)). Then for $j \geq s_{2},\left\{\mathbf{d}_{k}^{b} \mid \exists k\left[r_{j}\left(\mathbf{c}_{i}^{a}, \mathbf{d}_{k}^{b}\right)\right]\right\} \subseteq D_{j} \cup$ Free and $\left\{b \mid \exists k\left[r_{j}\left(\mathbf{c}_{i}^{a}, \mathbf{d}_{k}^{b}\right)\right]\right\} \subseteq B_{j}$, so that (A1) implies $\mathbf{c}_{i}^{a} \in D_{j}$. Thus $\mathbf{c}_{i}^{a} \in D$.

Finally since for all $y \in D_{i}$ and all $j \geq s_{2}, r_{j}\left(\mathbf{c}_{i}^{a}, y\right)$, Lemma 2 implies $D_{i} \subseteq D_{j}$ and hence $D_{i} \subseteq D$.

Corollary 3. $\left\{C_{i}\right\}_{i<\omega}$ and $\left\{D_{i}\right\}_{i<\omega}$ are $\Delta_{2}^{0}$ approximations to $C$ and $D$ respectively.

Proof. Towards a contradiction assume that $\left\{D_{i}\right\}_{i<\omega}$ is not a $\Delta_{2}^{0}$ approximation to $D$. Then there is an element $y \notin D$ such that the set $I(y)=\left\{i \mid y \in D_{i}\right\}$ is infinite. Every $i \in I(y)$ is a bad stage for $D$, and hence according to Proposition 7 it is a good stage for $A$. Since $I(y)$ is infinite,

$$
A=\left\{a \mid \exists i\left[i \in I(y) \& a \in A_{i}\right]\right\} .
$$

On the other hand $I(y)$ is computable and hence $A$ is c.e., contrary to what is given.

Similarly one proves that $\left\{C_{i}\right\}_{i<\omega}$ is a $\Delta_{2}^{0}$ approximation to $C$.

Corollary 4. $A=\left\{a \mid \exists j\left[\mathbf{c}_{j}^{a} \in C\right]\right\}$ and $B=\left\{b \mid \exists j\left[\mathbf{d}_{j}^{b} \in D\right]\right\}$. 
Proof. By Proposition 4 for every $i, A_{i}=\left\{a \mid \exists j\left[\mathbf{c}_{j}^{a} \in C_{i}\right]\right\}$ and $B_{i}=\left\{b \mid \exists j\left[\mathbf{d}_{j}^{b} \in\right.\right.$ $\left.\left.D_{i}\right]\right\}$. Hence if $a \notin A$, there is a stage $i_{a}$ such that $a \notin A_{i}$ for all $i>i_{a}$ and hence for all $j$ and all $i>i_{a}, \mathbf{c}_{j}^{a} \notin C_{i}$. This yields $A \supseteq\left\{a \mid \exists j\left[\mathbf{c}_{j}^{a} \in C\right]\right\}$.

Now let $a \in A$. Let $i_{a}$ be a stage such that $a \in A_{i}$ for all $i>i_{a}$. Let $j>i_{a}$ be a stage such that $B_{j} \nsubseteq B$. Such a stage exists, because if we assume otherwise, i.e. that for all $j>i_{a}, B_{j} \subseteq B$, it would follow that $B$ is c.e., contrary to what is given. At stage $j$, as $a \in A_{j}, \mathbf{c}_{j}^{a} \in C_{j}$ by (A2). By Proposition 7 as $B_{j} \nsubseteq B$, $C_{j} \subseteq C$. So $\mathbf{c}_{j}^{a} \in C$ and $A \subseteq\left\{a \mid \exists j\left[\mathbf{c}_{j}^{a} \in C\right]\right\}$. That $B=\left\{b \mid \exists j\left[\mathbf{d}_{j}^{b} \in D\right]\right\}$ is proved similarly.

To complete the verification of the construction in the last two propositions we prove that properties (P3) and (P4) are true.

Proposition 8. $D=\bar{C}$.

Proof. First we claim that $C \cap D=\emptyset$. Indeed, at each stage the rules of the construction guarantee the $C_{i} \cap D_{i}=\emptyset$. This together with the fact that $\left\{C_{i}\right\}_{i<\omega}$ and $\left\{D_{i}\right\}_{i<\omega}$ are $\Delta_{2}^{0}$ approximations implies $C \cap D=\emptyset$.

Next we prove that $C \cup D=\mathbb{N}$. Fix a natural $x \in \mathbb{N}$. Suppose that $x$ is not a follower. Without loss of generality we may assume that $x=\mathbf{c}_{i}^{a}$ for some natural numbers $i$ and $a$. Then at stage $s=\max \{i, a\}, x$ is enumerated in $D_{s}$ under rule (A3). It is never extracted from $D$. Indeed it could be extracted at a stage $j$ only under rule (E2), because this is the only rule which extracts an even number from $D$. However the set $\left\{\mathbf{d}_{k}^{b} \mid r_{j-1}\left(x, \mathbf{d}_{k}^{b}\right)\right\}=\emptyset$, so rule (E2) does not apply. Thus $x \in D$.

Now suppose that $x$ is a follower. If $x=\mathbf{c}_{i}^{a}$ for some $a \notin A$ or $x=\mathbf{d}_{i}^{b}$ for some $b \notin B$, then according to Proposition $7 x \in D$ or $x \in C$ respectively. So let $x=\mathbf{c}_{i}^{a}$ for some $a \in A$ and suppose that $x \notin C$. Then according to Proposition 7 for every $j$ if $x \in C_{j}$, then $B_{j} \subseteq B$. Thus if $r_{j}\left(x, \mathbf{d}_{k}^{b}\right)$, then $b \in B$. Let $s_{1}$ be the least stage such that for $j \geq s_{1}, x \notin C_{j}$. Then for $j \geq s_{1}$ we have $r_{j}\left(x, \mathbf{d}_{k}^{b}\right) \Longleftrightarrow r_{s_{1}}\left(x, \mathbf{d}_{k}^{b}\right)$. Furthermore Proposition 6 implies that for $j \geq s_{1},\left\{\mathbf{d}_{k}^{b} \mid r_{j}\left(x, \mathbf{d}_{k}^{b}\right)\right\} \cap C_{j}=\emptyset$ and hence $\left\{\mathbf{d}_{k}^{b} \mid r_{j}\left(x, \mathbf{d}_{k}^{b}\right)\right\} \subseteq D_{j} \cup$ Free. Let $s_{2} \geq s_{1}$ be the least stage such that for $j \geq s_{2},\left\{b \mid \exists k\left[r_{j}\left(x, \mathbf{d}_{k}^{b}\right)\right]\right\} \subseteq B_{j}$. Then at stage $s_{2}, x$ is enumerated in $D_{s_{2}}$ under rule (A1) and is never extracted from $D$.

Analogously we may prove that if $x=\mathbf{d}_{k}^{b}$ for some $b \in B$ and $x \notin D$, then $x \in C$.

Proposition 9. For every $i$, either $C_{i} \subseteq C$ or $D_{i} \subseteq D$.

Proof. Suppose that for some $i, C_{i} \not \subset C$ and let $x \in C_{i} \backslash C$. Fix a stage $s$ such that for each $j \geq s, x \in D_{j}$ (such a stage exists since $D=\bar{C}$ and the approximation to $D$ is $\left.\Delta_{2}^{0}\right)$. Take an arbitrary $y \in D_{i}$. Then for each $j \geq i, r_{j}(x, y)$, and hence according to claim (C1) of Lemma 2 we obtain that for $j \geq s, y \in D_{j}$. Thus $y \in D$ and hence $D_{i} \subseteq D$.

This completes the proof of Theorem 3 .

5.2. Weakly semi-recursive sets. The constructed maximal $\mathcal{K}$-pair is of the form $\{C, \bar{C}\}$, just as the ones from our initial examples, coming from a semi-recursive set and its complement. It would be natural to wonder if the sets produced by this construction are semi-recursive as well, and in that line of thought, if all $\mathcal{K}$ pairs of the form $\{C, \bar{C}\}$ consist of semi-recursive sets. In pursuit of this answer we 
come to a generalization of the notion of a semi-recursive set, weakly semi-recursive sets. Jockusch and Owings [10] have already defined this notion and used it in a completely different context - the theory of bounded queries.

Definition 8. We say that a set of natural numbers, $A$, is weakly semi-recursive if there is a computable selector function $s_{A}: \mathbb{N} \times \mathbb{N} \rightarrow \mathbb{N}$ such that: for any $x, y \in \mathbb{N}$, if $\{x, y\} \cap A \neq \emptyset$ and $\{x, y\} \cap \bar{A} \neq \emptyset$, then $s_{A}(x, y)$ is defined and $s_{A}(x, y) \in\{x, y\} \cap A$.

Every semi-recursive set is weakly semi-recursive. Furthermore every c.e. set is weakly semi-recursive. The selector function for a c.e. set works as follows: on every input $(x, y)$ it starts approximating the c.e. set until one of the arguments appears in the approximation and outputs this argument. It is not difficult to construct a c.e. set which is not semi-recursive: for every pair of elements $2 e$ and $2 e+1$, one waits until (if ever) the $e$-th partial computable function $\varphi_{e}$ is defined on argument $(2 e, 2 e+1)$ and diagonalizes against it by enumerating in the constructed set an element from $\{2 e, 2 e+1\} \backslash\left\{\varphi_{e}(2 e, 2 e+1)\right\}$.

Proposition 10. For every set of natural numbers $A$ the following are equivalent:

(1) $A$ is weakly semi-recursive.

(2) $\{A, \bar{A}\}$ form a $\mathcal{K}$-pair.

Proof. Suppose that $A$ is weakly semi-recursive with selector function $s_{A}$. Consider the set $W=\left\{\langle a, b\rangle \mid s_{A}(a, b) \downarrow=a\right\}$. Then $A \times \bar{A} \subseteq W$ and $\bar{A} \times A \subseteq \bar{W}$.

If on the other hand $\{A, \bar{A}\}$ form a $\mathcal{K}$-pair witnessed by $W$, then the graph of the selector function $s_{A}$ is $\{\langle\langle a, b\rangle, a\rangle \mid\langle a, b\rangle \in W\}$.

Finally we show that in this case as well, up to enumeration equivalence, all weakly semi-recursive sets, just as all semi-recursive sets, can be regarded as being of the form $L_{A}$.

Proposition 11. If $A$ is weakly semi-recursive, then $A \equiv_{e} L_{A}$.

Proof. By Proposition 1 we only need to show that $A \leq_{e} L_{A}$. Let $s_{A}$ be the selector function for $A$. Consider the enumeration operator $\Gamma$, defined as follows:

$$
\Gamma=\left\{\langle n, \sigma\rangle|| \sigma \mid=n+1 \& \sigma(n)=1 \& \forall m<n\left(\sigma(m)=0 \rightarrow s_{A}(n, m) \downarrow=n\right)\right\} .
$$

Then $A=\Gamma\left(L_{A}\right)$ can be seen as follows. If $n \in A$, then $\left\langle n, \chi_{A}\lceil(n+1)\rangle \in \Gamma\right.$ and $\chi_{A}\left\lceil(n+1) \in L_{A}\right.$. Suppose that $n \in \Gamma\left(L_{A}\right)$ via the pair $\langle n, \sigma\rangle \in \Gamma$. Towards a contradiction assume that $n \notin A$. We will show that every prefix $\tau \subset \sigma$ is an initial segment of $\chi_{A}$ by induction on its length. Suppose that for $\tau=\sigma \uparrow m$ we have that $\tau \subseteq \chi_{A}$. If $\tau * 1 \subseteq \sigma$, then as $\tau * 1 \in L_{A}$ and $\tau * 1$ is the rightmost extension of the rightmost string in $L_{A}$ of length $|\tau|, \tau * 1 \subseteq \chi_{A}$. If $\tau * 0 \subseteq \sigma$, i.e. $\sigma(m)=0$, then $s_{A}(n, m) \downarrow=n$ and as $n \notin A$ by the properties of a selector function $m \notin A$, $\tau * 0 \subseteq \chi_{A}$. Thus $\sigma \subseteq \chi_{A}$ and $\sigma(n)=1$ provides the anticipated contradiction.

Thus the statement of Theorem 3 can be further strengthened:

Corollary 5. For every nontrivial $\Delta_{2}^{0} \mathcal{K}$-pair $\{A, B\}$ there is a semi-recursive set $C$ such that $A \leq_{e} C$ and $B \leq_{e} \bar{C}$.

The class of nonzero enumeration degrees which contain $\Delta_{2}^{0}$ non-c.e. and nonco-c.e. semi-recursive sets is first order definable in $\mathcal{G}_{e}$. 
Proof. Let $\{A, B\}$ be a nontrivial $\mathcal{K}$-pair of $\Delta_{2}^{0}$ enumeration degrees. By Theorem 3 there is a $\Delta_{2}^{0} \mathcal{K}$-pair $\{C, \bar{C}\}$ such that $A \leq_{e} C$ and $B \leq_{e} \bar{C}$. By Proposition 10 the set $C$ is weakly semi-recursive. By Proposition 11 we have that $C \equiv_{e} L_{C}$. Thus $A \leq_{e} L_{C}$ and hence $B \leq_{e} \overline{L_{C}}=R_{C}$.

Thus a is the enumeration degree of a $\Delta_{2}^{0}$ non-c.e., non-co-c.e semi-recursive set $A$ if and only if $\mathbf{a}$ is half of a maximal $\Delta_{2}^{0} \mathcal{K}$-pair.

5.3. Relativization. Zooming out from the local structure and looking at the whole structure of the enumeration degrees, this is what we have so far.

From the definition of the enumeration jump of a set $A$, as $K_{A} \oplus \bar{K}_{A}$, it follows immediately that the jump of every enumeration degree is total. By Friedberg's Jump Inversion Theorem every total enumeration degree greater than or equal to $\mathbf{0}_{e}{ }^{\prime}$ belongs to the range of the enumeration jump operator. This together with the definability of the enumeration jump operator in $\mathcal{D}_{e}$ yields the first order definition of the total degrees above $\mathbf{0}_{e}{ }^{\prime}$. Thus by Theorem 4 the total degrees comparable to $\mathbf{0}_{e}{ }^{\prime}$ are first order definable in $\mathcal{D}_{e}$.

Furthermore the proof of Theorem 4 can be relativized above any total degree. Consider again the relativized version of a $\mathcal{K}$-pair: a pair of sets $A$ and $B$ forms a $\mathcal{K}$-pair over a set $U$ if there is a set $W \leq_{e} U$ such that

$$
A \times B \subseteq W \& \bar{A} \times \bar{B} \subseteq \bar{W} .
$$

Recall that this property is also degree theoretic and first order definable by

$$
\forall \mathbf{x}[\mathbf{x} \vee \mathbf{u}=(\mathbf{x} \vee \mathbf{a} \vee \mathbf{u}) \wedge(\mathbf{x} \vee \mathbf{b} \vee \mathbf{u})]
$$

In [7] we relativize the dynamic characterization of $\mathcal{K}$-pairs as follows:

Lemma 3 ([7). Let $G$ be a total set and let $B$ and $C$ be $\Sigma_{2}^{0}(G)$ sets. $B$ and $C$ form a $\mathcal{K}$-pair over $G$ if and only if $B$ and $C$ have $\Sigma_{2}^{0}(G)$ approximations $\left\{B_{i}\right\}_{i<\omega}$ and $\left\{C_{i}\right\}_{i<\omega}$ such that for every $i$ either $B_{i} \subseteq B$ or $C_{i} \subseteq C$.

Thus we can relativize the construction in the proof of Theorem 3 and obtain the following theorem:

Theorem 5. For every total enumeration degree a the class $\mathcal{T O} \mathcal{T} \cap\left[\mathbf{a}, \mathbf{a}^{\prime}\right]$ is first order definable in $\mathcal{D}_{e}$ with parameter $\mathbf{a}$.

\section{LOCAL DEFINABILITY OF THE LOW ENUMERATION DEGREES}

Now we turn to the local definability of the low enumeration degrees. The low enumeration degrees have been characterized in terms of the arithmetical complexity of the degrees that they bound. Cooper and McEvoy [4] show that an enumeration degree $\mathbf{a}$ is low if and only if every $\mathbf{b} \leq \mathbf{a}$ is $\Delta_{2}^{0}$. Giorgi, Sorbi and Yang [9] show that this characterization can be strengthened for the total enumeration degrees.

Definition 9. A $\Sigma_{2}^{0}$ set $A$ is called downwards properly $\Sigma_{2}^{0}$ if for every non-c.e. set $B$ such that $B \leq_{e} A, B$ is not $\Delta_{2}^{0}$. A degree a is downwards properly $\Sigma_{2}^{0}$ if it contains a downwards properly $\Sigma_{2}^{0}$ set.

Giorgi, Sorbi and Yang show that every nonlow total $\Sigma_{2}^{0}$ enumeration degree bounds a downwards properly $\Sigma_{2}^{0}$ enumeration degree.

In [6] we show that the class of downwards properly $\Sigma_{2}^{0}$ degrees is first order definable in $\mathcal{G}_{e}$. 
Theorem 6 ([6]). A $\Sigma_{2}^{0}$ degree is downwards properly $\Sigma_{2}^{0}$ if and only if it does not bound any nontrivial $\mathcal{K}$-pair.

Giorgi, Sorbi and Yang's result combined with the local first order definability of the classes of the total enumeration degrees and the downwards properly $\Sigma_{2}^{0}$ enumeration degrees already gives the first order definition of the low total enumeration degrees in $\mathcal{G}_{e}$. We could complete the proof of the definability of the low degrees simply by applying Soskov's Jump Inversion Theorem [23], which implies that a degree is low if and only if it is bounded by a low total degree.

To get a simpler definition we will first generalize the result by Giorgi, Sorbi and Yang.

Theorem 7. Every nonlow $\Sigma_{2}^{0}$ enumeration degree bounds a downwards properly $\Sigma_{2}^{0}$ enumeration degree.

Proof. Let $A$ be a member of a nonlow $\Sigma_{2}^{0}$ enumeration degree. Consider the $\mathcal{K}$-pair $L_{K_{A}}$ and $R_{K_{A}}$. Then by Proposition 11 $L_{K_{A}} \oplus R_{K_{A}} \equiv_{e} K_{A} \oplus \overline{K_{A}}=J_{e}(A)$. As $L_{K_{A}} \leq_{e} K_{A} \equiv_{e} A, L_{K_{A}}$ is a $\Sigma_{2}^{0}$ set. On the other hand, as $A$ is not low, $R_{K_{A}}$ is not a $\Sigma_{2}^{0}$ set, and so $L_{K_{A}}$ cannot be c.e. If we assume that $L_{K_{A}}$ is not downwards properly $\Sigma_{2}^{0}$, then by Theorem $\left[L_{K_{A}}\right.$ bounds a nontrivial $\mathcal{K}$-pair $\{C, D\}$. By the second property of Proposition 2. $\left\{C, D, R_{K_{A}}\right\}$ form a $\mathcal{K}$-triple with $C \oplus D \oplus R_{K_{A}} \not \leq_{e} \emptyset^{\prime}$. This contradicts Kalimullin's definition of the enumeration jump [12, which proves that $\mathbf{0}_{e}{ }^{\prime}$ is the largest enumeration degree which can be represented as a $\mathcal{K}$-triple. Thus $L_{K_{A}}$ is downwards properly $\Sigma_{2}^{0}$ and bounded by $A$.

Thus a degree is low if and only if it does not bound any downwards properly $\Sigma_{2}^{0}$ enumeration degree. Incorporating Theorem 6 this translates into a characterization of lowness in terms of the downwards density of $\mathcal{K}$-pairs: a degree is low if and only if every degree below it bounds a nontrivial $\mathcal{K}$-pair.

Theorem 8. The set of low enumeration degrees is first order definable in $\mathcal{G}_{e}$.

Proof. The low enumeration degrees are defined in $\mathcal{G}_{e}$ by the following formula:

$$
\mathcal{L} O W(\mathbf{x}) \Longleftrightarrow \forall \mathbf{b} \leq \mathbf{x} \exists \mathbf{c} \leq \mathbf{b} \exists \mathbf{d} \leq \mathbf{b}(\mathcal{L} K(\mathbf{c}, \mathbf{d})) .
$$

The definition of the total enumeration degrees below $\mathbf{0}_{e}{ }^{\prime}$ given in Theorem 4 , combined with the statement of Proposition 3, allows us to define the set

$$
C=\left\{\langle\mathbf{x}, \mathbf{u}\rangle \mid \mathbf{x}, \mathbf{u} \in \mathcal{T} \mathcal{O} \mathcal{T} \& \mathbf{u} \neq \mathbf{0}_{e} \& \iota^{-1}(\mathbf{x}) \text { c.e. in } \iota^{-1}(\mathbf{u})\right\} .
$$

Combining this with the definability of the low enumeration degrees, we could obtain a first order definition of the set of total enumeration degrees which are images of low Turing degrees, c.e. in some nonzero low Turing degree. Unfortunately the next theorem reveals that this set is not very interesting. It also gives a characterization of the $\Delta_{2}^{0}$ Turing degrees by an unexpected method.

Theorem 9. A Turing degree $\mathbf{x}$ is $\Delta_{2}^{0}$ if and only if $\mathbf{x}$ is c.e. in some low Turing degree.

Proof. One direction is obviously true: if $\mathbf{x}$ is c.e. in a low Turing degree, then $\mathbf{x}$ is $\Delta_{2}^{0}$. So let us concentrate on the opposite direction.

Suppose that $\mathbf{x}$ is a $\Delta_{2}^{0}$ Turing degree. If $\mathbf{x}$ is c.e., then it is c.e. in $\mathbf{0}_{T}$. Suppose that $\mathbf{x}=\mathbf{d}_{T}(X)$ is not c.e. Then the enumeration degree $\iota(\mathbf{x})=\mathbf{d}_{e}(X \oplus \bar{X})$ contains no $\Pi_{1}^{0}$ set. Consider the sets $L_{X}$ and $R_{X}$. It follows that neither of these sets is 
c.e., as otherwise $L_{X} \oplus R_{X}$ would be enumeration equivalent to a $\Pi_{1}^{0}$ set, and by Proposition $1 L_{A} \oplus R_{A} \equiv_{e} X \oplus \bar{X}$. Now we show that $L_{X}$ is low. To see this we use a familiar trick. As $K_{L_{X}} \equiv_{e} L_{X}$ it follows from Proposition 2 that $K_{L_{X}}$ forms a $\mathcal{K}$-pair with $R_{X}$, so $\overline{K_{L_{X}}} \leq_{e} R_{X} \oplus J_{e}(\emptyset)$. Now $J_{e}\left(L_{X}\right)=K_{L_{X}} \oplus \overline{K_{L_{X}}} \leq_{e}$ $L_{X} \oplus R_{X} \oplus J_{e}(\emptyset) \equiv_{e} J_{e}(\emptyset)$.

By Soskov's Jump Inversion Theorem 23 there is a low total degree $\iota(\mathbf{u})$ such that $\mathbf{d}_{e}\left(L_{X}\right) \leq \iota(\mathbf{u})$. Note that as $\iota$ preserves the jump operation, the Turing degree $\mathbf{u}$ is also low. So $\iota(\mathbf{x})$ is the least upper bound of a maximal $\mathcal{K}$-pair $\left\{\mathbf{d}_{e}\left(L_{X}\right), \mathbf{d}_{e}\left(R_{X}\right)\right\}$ such that $\mathbf{d}_{e}\left(L_{X}\right) \leq \iota(\mathbf{u})$. By Proposition 3 it follows that $\mathbf{x}$ is c.e. in $\mathbf{u}$.

\section{REFERENCES}

[1] M. M. Arslanov, I. Sh. Kalimullin, and S. B. Kuper, Splitting properties of total enumeration degrees (Russian, with Russian summary), Algebra Logika 42 (2003), no. 1, 3-25, 125, DOI 10.1023/A:1022660222520; English transl., Algebra Logic 42 (2003), no. 1, 1-13. MR.1988020 (2004e:03077)

[2] S. B. Cooper, Partial degrees and the density problem. II. The enumeration degrees of the $\Sigma_{2}$ sets are dense, J. Symbolic Logic 49 (1984), no. 2, 503-513, DOI 10.2307/2274181. MR745377 (85j:03068)

[3] S. Barry Cooper, Enumeration reducibility, nondeterministic computations and relative computability of partial functions, Recursion theory week (Oberwolfach, 1989), Lecture Notes in Math., vol. 1432, Springer, Berlin, 1990, pp. 57-110, DOI 10.1007/BFb0086114. MR1071514 (91i:03088)

[4] S. Barry Cooper and Kevin McEvoy, On minimal pairs of enumeration degrees, J. Symbolic Logic 50 (1985), no. 4, 983-1001 (1986), DOI 10.2307/2273985. MR820127 (87i:03086)

[5] Richard M. Friedberg and Hartley Rogers Jr., Reducibility and completeness for sets of integers, Z. Math. Logik Grundlagen Math. 5 (1959), 117-125. MR0112831 (22 \#3682)

[6] Hristo Ganchev and Mariya I. Soskova, Cupping and definability in the local structure of the enumeration degrees, J. Symbolic Logic 77 (2012), no. 1, 133-158, DOI $10.2178 / \mathrm{jsl} / 1327068696$. MR2951634

[7] Hristo Ganchev and Mariya Soskova, The high/low hierarchy in the local structure of the w-enumeration degrees, Ann. Pure Appl. Logic 163 (2012), no. 5, 547-566, DOI 10.1016/j.apal.2010.10.004. MR2880272(2012m:03104)

[8] Hristo Ganchev and Mariya Soskova, Interpreting true arithmetic in the local structure of the enumeration degrees, J. Symbolic Logic 77 (2012), no. 4, 1184-1194, DOI 10.2178/jsl.7704070. MR 3051620

[9] Matthew Giorgi, Andrea Sorbi, and Yue Yang, Properly $\Sigma_{2}^{0}$ enumeration degrees and the high/low hierarchy, J. Symbolic Logic 71 (2006), no. 4, 1125-1144, DOI 10.2178/jsl/1164060448. MR2275852 (2007i:03051)

[10] Carl G. Jockusch Jr. and James C. Owings Jr., Weakly semirecursive sets, J. Symbolic Logic 55 (1990), no. 2, 637-644, DOI 10.2307/2274653. MR1056377(91g:03086)

[11] Carl G. Jockusch Jr., Semirecursive sets and positive reducibility, Trans. Amer. Math. Soc. 131 (1968), 420-436. MR0220595 (36 \#3649)

[12] I. Sh. Kalimullin, Definability of the jump operator in the enumeration degrees, J. Math. Log. 3 (2003), no. 2, 257-267, DOI 10.1142/S0219061303000285. MR2030087 (2005d:03076)

[13] Alistair H. Lachlan and Richard A. Shore, The $n$-rea enumeration degrees are dense, Arch. Math. Logic 31 (1992), no. 4, 277-285, DOI 10.1007/BF01794984. MR1155038 (93b:03073)

[14] Kevin McEvoy, Jumps of quasiminimal enumeration degrees, J. Symbolic Logic 50 (1985), no. 3, 839-848, DOI 10.2307/2274335. MR805690(87m:03060)

[15] André Nies, Richard A. Shore, and Theodore A. Slaman, Interpretability and definability in the recursively enumerable degrees, Proc. London Math. Soc. (3) 77 (1998), no. 2, 241-291, DOI 10.1112/S002461159800046X. MR.1635141(99m:03083)

[16] Hartley Rogers Jr., Theory of recursive functions and effective computability, McGraw-Hill Book Co., New York, 1967. MR0224462 (37 \#61) 
[17] M. Rozinas, The semilattice of e-degrees (Russian), Recursive functions (Russian), Ivanov. Gos. Univ., Ivanovo, 1978, pp. 71-84. MR604944(82i:03057)

[18] R. A. Shore, Biinterpretability up to double jump in the degrees below $\mathbf{0}^{\prime}$, Proc. Amer. Math. Soc. 142 (2014), no. 1, 351-360. MR.3119208

[19] Richard A. Shore, Degree structures: local and global investigations, Bull. Symbolic Logic 12 (2006), no. 3, 369-389. MR2248589 (2007e:03074)

[20] Richard A. Shore and Theodore A. Slaman, Defining the Turing jump, Math. Res. Lett. 6 (1999), no. 5-6, 711-722, DOI 10.4310/MRL.1999.v6.n6.a10. MR1739227 (2000m:03104)

[21] Theodore A. Slaman, Global properties of the Turing degrees and the Turing jump, Computational prospects of infinity. Part I. Tutorials, Lect. Notes Ser. Inst. Math. Sci. Natl. Univ. Singap., vol. 14, World Sci. Publ., Hackensack, NJ, 2008, pp. 83-101, DOI 10.1142/9789812794055_0002. MR2449478 (2009k:03065)

[22] Andrea Sorbi, The enumeration degrees of the $\Sigma_{2}^{U}$ sets, Complexity, logic, and recursion theory, Lecture Notes in Pure and Appl. Math., vol. 187, Dekker, New York, 1997, pp. 303330. MR 1455141 (98g:03107)

[23] I. N. Soskov, A jump inversion theorem for the enumeration jump, Arch. Math. Logic 39 (2000), no. 6, 417-437, DOI 10.1007/s001530050156. MR1773778 (2001g:03078)

Faculty of Mathematics and Informatics, Sofia University, 1164 Sofia, Bulgaria

E-mail address: ganchev@fmi.uni-sofia.bg

Department of Mathematics, University of California Berkeley, Berkeley, CaliforNIA 94720

Current address: Faculty of Mathematics and Informatics, Sofia University, 1164 Sofia, Bulgaria

E-mail address: msoskova@fmi.uni-sofia.bg 